\title{
PRECIOS Y PRODUCTO EN EL CORTO PLAZO: ENFOQUES TEÓRICOS ALTERNATIVOS
}

\author{
Alain Ize \\ El Colegio de México

\section{FORMACIÓN DE PRECIOS Y DEL PRODUCTO: ALGUNAS DE LAS GRANDES PREGUNTAS.} \\ LAS TEORÍAS PROPUESTAS HASTA AHORA para explicar la formación de precios \\ y del producto pueden ser ubicadas en forma general dentro de dos grandes para- \\ digmas; el primero podría denominarse neoclásico, el otro, que reagrupa teorías \\ como la marxista o las estructuralistas, a falta de un nombre más apropiado, \\ podría llamarse el paradigma sociológico. Cada uno de estos grandes cuerpos de \\ teorías ha sido desarrollado para tratar de contestar ciertos tipos de interrogantes \\ fundamentales que serán reseñados brevemente a continuación.
}

\section{Del equilibrio al desequilibrio.}

Dentro del paradigma neoclásico, la explicación y la predicción de los cambios en los niveles de precios y en el producto agregado,-problema central en el análisis macroeconómico de corto plazo-es un tema que se ha desarrollado en forma un tanto pendular desde principios de siglo. El nivel de análisis se ha hecho cada vez más refinado y complejo pero ha alternado entre los mismos dos polos de pensamiento: por un lado, la idea de una economía de mercado como un sistema autoregulable y que sabe mantenerse siempre en equilibrio; y por otro lado, la visión de un sistema en el cual la falta de mecanismos autoreguladores y el impacto de choques incesantes conduce a la existencia casi permanente de estados de desequilibrio.

La macroeconomía "clásica" de principios de siglo asociada con los nombres de Pigou, Fisher y Marshall, constituye un primer ejemplo de teoría del equilibrio: ofrece la visión de una economía permanentemente en el pleno empleo y en donde el dinero es perfectamente neutral: ${ }^{1}$ no altera el nivel "real" de actividad económica sino que fija solamente (y en exclusiva) el nivel absoluto de los precios. La inflación es, por lo tanto, un fenómeno monetario, y su control -preocupación central de la época-pasa por el control adecuado de la oferta monetaria.

Keynes y la gran depresión vinieron a alterar radicalmente la ortodoxia clásica

1 Véase Pigou (1933), Fisher (1911) y Marshall (1920). 
hasta entonces prevaleciente. ${ }^{2}$ Keynes, en su teoría general, ${ }^{3}$ propuso la idea de un sistema fundamentalmente en desequilibrio, que puede permanecer persistentemente por debajo del nivel de pleno empleo y en el cual la cantidad de dinero puede tener un impacto sobre el nivel de actividad económica más que sobre los precios. Introdujo también el concepto novedoso de un estado siempre activo que busca, a través de sus políticas fiscales y monetarias, suplir las deficiencias de los mercados y mantener la economía cerca de su equilibrio de pleno empleo.

La teoría Keynesiana, ampliamente aplicada después de la guerra, llegó a convertirse en la nueva ortodoxia, al mismo tiempo que era formalizada e integrada dentro de esquemas y corrientes económicas más tradicionales. Esta labor de reconstrucción y formalización, asociada con los nombres de economistas como Hicks, Hansen y Patinkin, ${ }^{4}$ culminó en lo que se conoce como la "síntesis neoclásica", que permite contrastar en forma clara las teorías clásicas y keynesianas: la diferencia esencial entre ambas es la hipótesis que se quiera hacer sobre la flexibilidad de los salarios: perfectamente flexibles en los clásicos, rígidos hacia abajo en la teoría Keynesiana.

Keynes no dio, sin embargo, una definición clara de la relación precios/producto en su modelo. ${ }^{5}$ En los modelos macroeconómicos de libros de texto, esta carencia fue inicialmente resuelta mediante la introducción de una dicotomía completa entre movimientos en precios y en cantidades: por debajo del pleno empleo, los precios son rígidos y las cantidades, flexibles; al llegar al pleno empleo, las cantidades se vuelven rígidas y todo intento de estimulación adicional se evapora en alzas de precios. Esta dicotomía aguda fue suavizada posteriormente con la introducción de la curva de Phillips. ${ }^{6}$ Según este aporte existe, alrededor del pleno empleo, una relación estable entre inflación y producto, y el gobierno puede escoger la combinación de estas dos variables que más le convenga.

Luego de tres o cuatro décadas de relativo éxito en mantener niveles de empleo relativamente altos, el recuerdo de la gran depresión se fue borrando y con ello fue disminuyendo el interés por la teoría Keynesiana. En cambio las preocupaciones se volcaron progresivamente hacia las tasas de inflación cada vez mayores que experimentaban la mayoría de las economías de mercado y que las teorías Keynesianas no lograban explicar en forma satisfactoria. Este modelo empezó entonces a ser criticado con una fuerza creciente y llegó finalmente a ser reemplazado casi totalmente por una nueva ortodoxia, la de los llamados "nuevos clásicos". La contra-revolución empezó con una crítica severa a la curva de Phillips, por parte de Phelps y Friedman. ${ }^{7}$ Ellos argumentaron que la existencia de una curva de Phillips estable significaba que los agentes económicos podían ser engañados perrnanentemente, lo que no es permisible en un universo en el cual se supone que existe un mínimo de "racionalidad". En el largo plazo, el gobierno no pue-

2 El nombre de Kalecki deberia, en toda justicia, estar tambien asociado a esta revolución en el pensamiento económico; véase en particular Kalecki (1954).

3 Véase Keynes (1936).

4 Véase Hicks (1937), Hansen (1953) y Patinkin (1956).

5 Esta relación es la llamada "ecuación faltante" de la teoría keynesiana.

6 Véase Phillips (1958) y Lipsey (1960).

7 Véase Phelps (1967 y 1970) y Friedman (1968). 
de, por lo tanto, empujar la economía más allá de su nivel "natural" de pleno empleo.

Las nuevas ideas se centraron en el análisis de una economía en la cual pueden darse fallas de información puesto que la transmisión de la información es costosa e imperfecta. La integración de las fallas de información y del concepto de "expectativas racionales" dentro del molde del modelo clásico forma la esencia de esta contra-revolución cuyo desarrollo está asociado con los nombres de economistas como Muth, Lucas, Sargent y Wallace. ${ }^{8}$ Según esta corriente, las fluctuaciones en el ingreso se deben solamente a errores de percepción por parte de los agentes más no a la permanencia de estados de desequilibrio en los mercados. El pensamiento económico ha dado así una vuelta casi completa, al regresar muy cerca de su punto de partida: un sistema económico que es fundamentalmente estable y permanece en equilibrio; se integra ahora además al modelo el concepto novedoso de información imperfecta.

Paralelamente a esta contra-revolución, se siguió sin embargo el esfuerzo de reconstrucción y de síntesis de las teorías keynesianas y pre-keynesianas. El objetivo perseguido consistió en modelar las características esenciales del esquema keynesiano dentro del molde walrasiano que hasta ese momento era la exclusividad de los modelos de equilibrio general a la Arrow-Debreu. Los aportes de Clower y Leijonhufvud, ${ }^{9}$ hacia fines del sesenta, ${ }^{10}$ fueron determinantes para impulsar la modelación de sistemas económicos con precios totalmente rígidos y mercados en desequilibrio. Esta corriente de pensamiento, que fue posteriormente refinada en Francia por economistas como Benassy y Malinvaud, ${ }^{11}$ constituye quizás en la actualidad el ramal más elaborado y más prometedor de la teoría keynesiana. ${ }^{12}$

La controversia en torno a los modelos de equilibrio versus los de desequilibrio está ahora culminando y no parece haber llegado aún a una conclusión clara y satisfactoria. ${ }^{13}$ Sobre todo porque no ha sido formulada aún una teoría realmente convincente y general que permita explicar rigideces en precios y salarios y que proponga leyes y dinámicas de ajuste adecuadas. Quizás haya que buscar de hecho algunos elementos de esta teoría dentro de paradigmas alternativos que enfaticen los aspectos más "estructurales" o "sociológicos" de la realidad económica. Estos enfoques son precisamente los que se reseñan brevemente a continuación.

8 Véase Muth (1961), Lucas (1972) y Sargent y Wallace (1973 y 1975 y las reseñas en McCallum (1980), Kantor (1979), Barro (1979) y Lucas y Sargent (1980).

9 Véase Clower (1965), Leijonhufvud (1968) y también Barro y Grossman (1971).

10 Habria que ligar, de hecho, estos trabajos con los de Patınkin que fue el primero en modelar el concepto de "equilibrio bajo restricciones".

11 Véase Benassy (1975) y Malinvaud (1977) y las teseñas en Benassy (1980) y Drazen (1980).

12 Esta opinión no es, sin embargo, compartida en algunos medios keynesianos "fundamentalistas" anglo-sajones, de ambos lados del Atlántico. Véase por ejemplo, Kahn (1977), Davidson (1972) y Weintraub (1978).

13 La literatura en este sentido es cada día más extensa. Véase, por ejemplo, Ando (1978) y Modigliani (1977), Solow (1980), Hahn (1980), Tobin (1981) y Gordon (1981), por un lado; Barro (1979a), McCallum (1979 y 1980) y Lucas (1981), por el otro. 


\section{De los choques de oferta a la crisis de acumulación}

El común denominador de las teorías pertenecientes al paradigma sociológico es el énfasis puesto sobre la distribución del producto entre los diferentes sectores o categorías sociales. En vez de asociar el proceso inflacionario con una creación excesiva de dinero que viene a perturbar un sistema que en su esencia profunda es armónico y estable, estas teorías establecen una estrecha relación entre inflación y los conflictos internos que surgen alrededor de la distribución del producto: hay inflación a partir del momento en que las peticiones de participación en el ingreso de las diferentes categorías sociales rebasan la capacidad productiva de la economía; el alza de precios permite así armonizar, "ex-post", las inconsistencias que se presentan "ex-ante" entre el tamaño del ingreso total y las demandas de todos los sectores.

Esta idea central aparece en varios escritos de los principios de los años $50,{ }^{14}$ aunque su germen sea quizá más antiguo. Fue posteriormente refinada y adaptada dentro de varios marcos de análisis más específicos.

El marco más sencillo es el que relaciona la espiral inflacionaria con el exceso de demandas salariales impuesto por los sindicatos. Varios autores analizan esta hipótesis e intentan medir el grado de asociación que existe entre inflación y militancia sindical, sin lograr sin embargo poner en evidencia una clara relación, ${ }^{15}$ quizás, en parte, por la dificultad existente en obtener un buen indice de militancia.

Un marco un poco más elaborado es el que relaciona las peticiones de un grupo social con las de otro grupo, por ejemplo, las de trabajadores en sectores con bajo crecimiento de productividad con las de sus colegas en sectores con fuertes alzas de productividad. Esto redunda en inflación porque las alzas salariales acordadas en los sectores muy productivos son excesivas en relación con los aumentos de productividad en los sectores más atrasados. Este mecanismo de inflación estructural parece tener un buen grado de relevancia empírica y ha sido analizado por numerosos autores. ${ }^{16}$

Otro mecanismo inflacionario "estructuralista", que ha sido asociado con las escuelas latinoamericanas, es el que parte de las deficiencias de oferta que afectan la producción agrícola. Frente a una demanda de bienes de consumo alimenticio relativamente inelástica, estos problemas de oferta repercuten en alzas de los precios agrícolas. Estas alzas son a su vez transmitidas rápidamente al resto de la economía porque los agentes económicos tratan de defender sus ingresos mediante incrementos proporcionales del precio de venta de sus productos,

14 Véase por ejemplo Aujac (1950), Le Bourva (1950), Holzman (1950), Morton (1950), Bronfenbrenner (1950), Ackley (1959), Thorp y Quandt (1959) y la reseña en Bronfenbrenner y Holzman (1963).

15 El primer autor que trató de medir esta asociación es Hines (1964). Numerosos estudios lo siguieron. Véase para este efecto la reseña en Laidler y Parkin (1975) pp. 761-765. Un estudio reciente sobre este tema es el de Spinelli (1981).

16 Véase en particular Brechling (1973), Mulvey y Trevithick (1974), Eatwell, Llewellyn y Tarling (1974) y la reseña en Laidler y Parkin (1975). Un mecanismo bastante similar de inflación estructural basada sobre diferenciales de demanda sectorial y ajustes salariales uniformes fue también propuesta en Estados Unidos por economistas como Schultza (1959). 
ya sean bienes o trabajo. ${ }^{17}$ Los trabajos estructuralistas enfatizan generalmente las deficiencias productivas endémicas del sector agrícola, lo que permite justificar la existencia de un proceso inflacionario persistente. Pero el mismo esquema sirve también para explicar brotes inflacionarios transitorios producidos por choques de oferta de cualquier otra naturaleza.

Al lado de estas teorías, existen también otras interpretaciones del proceso inflacionario que están más directamente ligadas al proceso de acumulación de las economías capitalistas. Una de éstas es la que se desarrolló en Cambridge y que por su énfasis en las expectativas de crecimiento como variable exógena, se conoce generalmente como neo-keynesiana. Según esta interpretación, la inflación es e 1 medio que usan las empresas para reducir el sueldo real de los trabajadores y poder así generar las ganancias necesarias para satisfacer los requerimientos de inversión propios a la tasa de crecimiento esperada por los empresarios. El estado, al aumentar paralelamente la oferta monetaria, se hace implícitamente cómplice de los capitalistas en su búsqueda por mayores utilidades. ${ }^{18}$

La otra teoría centrada sobre la acumulación es la que se podría clasificar como neo-marxista por el papel central que juegan las contradicciones asociadas con las luchas de clases. Enfatizan, más en particular, el rol del estado que trata, a través del manejo del gasto público, de aliviar las tensiones y conflictos sociales propios a la acumulación capitalista. Estos intentos redundan, sin embargo, en el crecimiento exagerado de un sector público poco productivo, hecho que, al frenar la capacidad de oferta en relación con el nivel de las peticiones sociales, tiende a crear presiones inflacionarias persistentes. ${ }^{19} \mathrm{La}$ crítica inmediata que se le puede hacer, sin embargo, a esta teoría es que no permite explicar fácilmente el por qué de las grandes variaciones en las tasas de inflación que ocurren año tras año en todas las economías de mercado. Parecería necesario introducir, para tal efecto, algún tipo de choques o acontecimientos estocásticos adicionales alrededor de los cuales se pudieran cristalizar los conflictos sociales.

Una de las principales preguntas que cabe plantearse dentro de estos enfoques sociológicos quizás sea precisamente la siguiente: ¿deberá de interpretarse la inflación como un proceso de largo plazo ligado a una inconsistencia o contradicción endémica del sistema económico y social? ¿O será simplemente el resultado de discrepancias temporales entre la productividad y las peticiones sociales causadas por choques estocásticos de oferta y rigideces en la estructura de las peticiones? Estas preguntas vienen a sumarse a las que ya fueron formuladas anteriormente dentro del universo económico tradicional y se suman también a la pregunta, aún más fundamental, que concierne a la selección misma del paradigma más apropiado de estudio.

17 El estructuralismo latinoamericano parece haber surgido en la década del cincuenta. Algunas de las contribuciones más importantes de esta corriente de pensamiento son las de Noyola (1956), Seers (1962), Olivera (1964) y Prebisch (1970). Véanse también las reseñas en Wachter (1976) y Solís (1971).

18 Véase en particular Kaldor (1959) y Robinson (1973).

19 Algunos de los trabajos más representativos dentro de este enfoque son los de Rowthorn (1980) y Boyer y Mistral (1978). Véase también la reseña en Harvey (1977). Una interpretación no marxista del mismo fenómeno puede también ser encontrada en Soderstrom y Viotti (1975). 


\section{La transición a una economía abierta.}

La ampliación al caso de una economía abierta de las teorías reseñadas hasta ahora no se hizo esperar mucho; siguió de cerca los desarrollos alcanzados para economías cerradas y fue alternando con ellos entre los diferentes paradigmas.

Después de la teoría general, el modelo "ortodoxo" de determinación del producto y de la balanza de pagos era esencialmente keynesiano, dado que partía de un esquema de precios y salarios rígidos junto con excesos de oferta generalizados. $^{20}$ En este modelo, el único precio variable es la tasa de cambio que es controlada por el gobierno. Y el objetivo esencial de política consiste en alcanzar cierto nivel de producción interna y cierto nivel de equilibrio en la cuenta corriente. La combinación de estos dos objetivos se logra mediante ajustes simultáneos del gasto público y de la tasa de cambio, siendo una devaluación benéfica para la cuenta corriente a partir del momento en que se verifica cierta condición sobre las elasticidades de las funciones de importaciones y exportaciones. Si el punto inicial es de pleno empleo, una devaluación, para ser efectiva, requiere además de una reducción en el gasto público que logre reducir la demanda interna (o "absorción" interna) lo suficiente como para liberar el residuo exportable necesario.

Este modelo empezó, sin embargo, a ser criticado desde los años sesenta y fue paulatinamente descartado a raíz del resurgimiento monetarista que cuestionó la hipótesis básica de rigidez en los precios y salarios. Al eliminar esta hipótesis, surgió el llamado "enfoque monetario de la balanza de pagos" según el cual, tanto el producto como el déficit en cuenta corriente y los precios, son funciones exclusivas de la cantidad de dinero existente en la economía. Las hipótesis de información imperfecta, errores de percepción y expectativas racionales de los "nuevos clásicos" fueron incorporadas posteriormente a este modelo, convirtiéndolo en el enfoque contemporáneo dominante. ${ }^{21}$

Recientemente, sin embargo, ha surgido también bastante interés en modelar rigurosamente características keynesianas de rigideces en precios y salarios dentro del marco más elaborado del nuevo modelo monetarista, volviéndose así a encontrar resultados más parecidos a los que había aportado la primera generación de modelos keynesianos. ${ }^{22}$

Finalmente, han aparecido también en años recientes, un buen número de modelos que tratan de apartarse del supuesto neoclásico de competencia perfecta y examinar el comportamiento de una economía abierta en la cual las empresas fijan sus márgenes de ganancia y los trabajadores su sueldo real. Una de las versiones más populares es la del modelo "estructuralista escandinavo", según el cual los trabajadores de los sectores que producen bienes no comerciables exigen aumentos de sueldo similares a los que obtuvieron los trabajadores de los sectores

20 Este enfoque remonta a Robinson (1937), Machlup (1939), Haberler (1949) y Meade (1951). Fue desarrollado posteriormente por autores como Swan (1955) y Mundell (1961).

21 Véanse los aportes originales de Mundell (1968), Dornbusch (1973) y Frenkel y Johnson (1976). La generalización al paradigma de información imperfecta de la nueva escuela clásica se debe en particular a Black (1973), Kouri (1975) y Dornbusch (1980).

22 Véase por ejemplo Dornbusch (1979), Dixit (1978) y Negishi (1979). 
de comerciables; sin embargo, los aumentos de productividad del sector "expuesto" de comerciables siendo mayores a los del sector de no comerciables; esto da origen a una incompatibilidad entre peticiones y productividad, similar a las que ya habían sido encontradas anteriormente en el caso de una economía cerrada; lo que genera a su vez un proceso inflacionario persistente. ${ }^{23}$ Otra versión, dentro de este mismo enfoque sociológico, enfatiza por su parte el papel del déficit en cuenta corriente y del nivel de empleo como variables adicionales de ajuste para armonizar peticiones y productividad. ${ }^{24}$

\section{MODELOS ALTERNATIVOS EN UNA ECONOMÍA CERRADA}

\section{Caracteristicas generales.}

Se desarrollarán ahora, en forma muy resumida, algunos modelos característicos de formación de precios y del producto en el corto plazo, con el fin de asociar mejor las hipótesis básicas que los sustentan con sus implicaciones de comportamiento y de política. Para fines de simplificación, se considerará una economía con un solo activo financiero que no deviene intereses y cuya demanda es insensible a los cambios en la tasa de inflación. Este activo, emitido por el gobierno, se define como dinero. Se considerarán solamente un insumo homogéneo, trabajo, y un producto. Dado el énfasis puesto sobre el corto plazo, el acervo de capital físico se tomará como dado y no será incluido en los modelos. Se supondrá también, para empezar, que la economía está cerrada, relajándose posteriormente esta hipótesis.

La notación será la que sigue:

$$
\begin{array}{ll}
M & : \text { oferta de dinero } \\
M^{D} & : \text { demanda de dinero } \\
L^{\mathrm{O}} & : \text { oferta de trabajo } \\
L^{D} & : \text { demanda de trabajo } \\
Y & : \text { producto } \\
P & : \text { precio del bien } \\
P^{e} & : \text { precio esperado del bien } \\
W & : \text { salario. }
\end{array}
$$

En los modelos de economía abierta que se desarrollarán a continuación, se usarán letras minúsculas en vez de mayúsculas indicando, entonces, que las magnitudes correspondientes son logaritmicas.

23 Los trabajos más conocidos dentro del enfoque estructuralista escandinavo son los de Aukrust (1970), Branson y Myhrman (1976) y Maynard y Rijckeghem (1976).

24 Véase, por ejemplo, Soderstrom y Viotti (1975), Cardoso (1979), Ize (1980) y las contribuciones de la "nueva" escuela de Cambridge, por ejemplo, las de Fetherston y Godley (1978 y 1979). 


\section{El modelo clásico tradicional.}

Dos hipótesis básicas sustentan el modelo clásico: por una parte, una hipótesis de convexidad estricta de los conjuntos tecnológicos y de preferencia; por otra parte, una difusión perfecta de información sobre las tecnologías, las características de los bienes y sus precios. Este conjunto de hipótesis conduce a una economía en la cual no existe ningún tipo de barreras a la entrada para la producción y los mercados son perfectos. Esto a su vez implica que existe competencia perfecta y que los precios son determinados libremente por la acción de los mercados, de tal forma que se igualen siempre instantáneamente ofertas y demandas. Estos supuestos se traducen e.l un modelo del tipo siguiente:

$$
\begin{aligned}
& y=\alpha l^{D}+\beta, \alpha<1 \\
& l^{D}=\alpha^{\prime}(p-w)+\beta ! \alpha^{\prime}>0 \\
& l^{O}=\alpha^{\prime \prime}(w-p)+\beta^{\prime \prime}, \alpha^{\prime \prime}>0 \\
& m^{D}=p+\gamma y+\nu, \gamma>0 \\
& m^{D}=m \\
& l^{D}=l^{O}
\end{aligned}
$$

La ecuación (1) es una función de producción con retornos decrecientes en el uso del factor trabajo; (2) es la demanda de trabajo que se deriva de la maximización de ganancias de las empresas en un contexto perfectamente competitivo (producto marginal igual al salario real); ${ }^{25}$ (3) es la oferta de trabajo que se deriva de una maximización de utilidades individuales, tomando consumo y ocio como variables; (4) es la ecuación de demanda de dinero, que, en ausencia de ilusión monetaria, se supone homogénea en dinero y precios. ${ }^{26}$ Finalmente, (5) y (6) son las condiciones de equilibrio en los mercados de dinero y de trabajo.

La resolución de este modelo es inmediata. El equilibrio en el mercado de trabajo [ecuaciones (2), (3) y (6)] define una cantidad de trabajo y un sueldo real de equilibrio:

$$
w^{*}-p^{*}=\frac{\beta^{\prime}-\beta^{\prime \prime}}{\alpha^{\prime}+\alpha^{\prime \prime}}
$$

25 Dada esta relación, $\alpha^{\prime}$ y $\beta^{\prime}$ se derivan de $\alpha$ y $\beta$. La forma específica de esta relación no es particularmente relevante para el desarrollo del modelo.

26 Dado que existe en este modelo un solo activo financiero, la demanda del bien se deduce de la demanda de dinero: lo que no se gasta se guarda en forma de dinero y viceversa. Esto simplifica el modelo ya que permite no tener que escribir una demanda separada del bien (la curva IS). 
La función de producción define a su vez el valor del producto:

$$
y^{*}=\frac{\alpha \alpha^{\prime}\left(\beta^{\prime}+\beta^{\prime}\right)+\beta}{\alpha^{\prime}+\alpha^{\prime \prime}}
$$

Finalmente, la condición de equilibrio en el mercado monetario y la demanda de dinero definen el nivel de precios:

$$
p^{*}=m-\gamma y^{*}-v
$$

Estas tres últimas ecuaciones resumen bien las características del modelo clásico: el sueldo real y el producto tienen un valor de equilibrio único que no depende del dinero, sino solamente de los parámetros "reales" de la economía, derivados de las preferencias de los consumidores y de la tecnología. El nivel absoluto de precios es a su vez simplemente proporcional a la cantidad de dinero y la inflación es por lo tanto un fenómeno esencialmente monetario: habrá alzas de precios cada vez que la oferta monetaria aumente más rápidamente que el producto.

Las implicaciones de política son claras: los niveles del producto y de empleo no son alterables mediante el manejo del gasto público ${ }^{27}$ y el papel del gobierno se limita a hacer crecer moderadamente la oferta monetaria para evitar presiones inflacionarias excesivas. De este modelo se deriva una filosofía de no intervención y disciplina monetaria que forma el núcleo de las recomendaciones monetaristas.

\section{Los nuevos clásicos}

Como se vio anteriormente, la simplicidad y claridad del modelo clásico descansa sobre dos supuestos bastante restrictivos: los de convexidad estricta e información perfecta. La pregunta que surge inmediatamente es la siguiente: ¿qué pasaría si se abandonaran alguno de estos supuestos o ambos?

El supuesto que relajan los "nuevos clásicos" es el segundo, pero en forma un tanto especial. Suponen, en primer lugar, que el universo dentro del cual se ubica la economía es estocástico, de tal forma que choques de oferta y de demanda vienen constantemente a alterar la función de producción y la demanda de dinero. Suponen, también, que por causa de algunas deficiencias en la información tanto los agentes económicos como el gobierno tardan en conocer la magnitud de estos choques. ${ }^{28}$ Suponen finalmente que las empresas y los trabajadores tienen acceso diferencial a la información sobre el valor actual de equilibrio del

27 En este modelo, puesto que no hay impuestos ni bonos gubernamentales, no hay una separación entre la política monetaria y la política fiscal; un aumento del gasto tiene necesariamente que producirse vía en aumento de la oferta monetaria.

28 Se podría también suponer que tardan en reaccionar a estos choques, aunque los conozcan inmediatamente. Bajo ciertas condiciones de igual tardanza por parte del gobierno y del resto de los agentes económicos, se encontraría un modelo con características similares al de información imperfecta. Pero los costos de ajuste necesarios para justificar estos atrasos se integrarán de preferencia dentro del modelo keynesiano. 
precio del bien: las empresas, por su contacto directo con el mercado, lo conocen inmediatamente; los trabajadores sólo con cierto atraso. Dados estos supuestos, el modelo clásico presentado en (1) - (6) se reescribe como sigue:

$$
\begin{aligned}
y^{O} & =\alpha l^{D}+\beta+\epsilon^{O}, \alpha<1 \\
l^{D} & =\alpha^{\prime}(p-w)+\beta^{\prime}, \alpha^{\prime}>0 \\
l^{O} & =\alpha^{\prime \prime}\left(w-p^{e}\right)+\beta^{\prime \prime}, \alpha^{\prime \prime}>0 \\
m^{D} & =p+\gamma y+v+\epsilon^{D}, \gamma>0 \\
m^{D} & =m \\
l^{D} & =l^{O}
\end{aligned}
$$

El conjunto de ecuaciones es el mismo. Se reemplaza solamente $p$ por $p^{\mathbf{e}}$ en la ecuación de oferta de trabajo y se añaden dos términos estocásticos, $\epsilon^{\boldsymbol{O}}$ y $\epsilon^{\boldsymbol{D}}$, que representan los choques de oferta y demanda. Se supone, para mayor simplicidad, que estos errores son "ruido blanco", o sea que son distribuidos normalmente con media cero y sin ninguna correlación serial. ${ }^{29}$

La resolución de este modelo sigue los mismos pasos que en el caso anterior, sólo que ahora la oferta del bien se escribe:

$$
y=y^{*}+\frac{\alpha \alpha^{\prime} \alpha^{\prime \prime}}{\alpha^{\prime}+\alpha^{\prime \prime}}\left(p-p^{e}\right)+\epsilon^{o}
$$

en donde $y^{*}$ es el nivel de equilibrio del modelo clásico. Y con esta última relación, la ecuación de demanda de dinero conduce a una expresión del tipo siguiente: ${ }^{30}$

$$
p=a m+(l-a) p^{e}-a\left(\gamma y^{*}+v\right)-a\left(\epsilon^{D}+\gamma \epsilon^{O}\right)
$$

De las ecuaciones (16) y (17), se aprecia que, para un valor dado del precio esperado $p^{\mathrm{e}}$, existen relaciones positivas entre el precio, el ingreso y la oferta monetaria, de tal forma que es posible elevar el ingreso vía estímulos monetarios, aunque se eleve simultáneamente el nivel de precios. Esto es en esencia lo que expresa la curva de Phillips de los años sesenta.

La contribución básica de los nuevos clásicos fue de señalar que era sin embargo irracional tomar las expectativas de precios como fijas. Argumentaron en cambio, en forma bastante convincente, que los agentes económicos deberían de

29 Se podrían considerar distribuciones más generales (por ejemplo, con algún tipo de correlación serial) pero esto no cambiaría los resultados si los agentes conocen las características exactas de estas distribuciones.

$30 a$ es una constante cuyo valor es:

$$
a=1 /\left(1+\frac{\left.\gamma \alpha \alpha^{\prime} \alpha^{\prime \prime}\right)}{\alpha^{\prime}+\alpha^{\prime \prime}}\right.
$$


ajustar eventualmente sus expectativas hacia el valor real observado, de tal forma que la diferencia entre $p$ y $p^{e}$ no mostrara una correlación serial importante, lo que reflejaría errores de percepción sistemáticos $\mathrm{y}$, por tanto, alguna irracionalidad. Es claro entonces que cuando los agentes forman sus expectativas racionalmente, cesa de existir para el gobiemo una relación durable entre precios y producto: cualquier intento de estimular el producto será exitoso inicialmente, en la medida en que logre sorprender al público e inducirlo en el error; pero cesará de serlo en cuanto los agentes se den cuenta del error cometido y rectifiquen sus expectativas. Según la expresión ya bien conocida, esto se traduce por una absoluta "verticalidad" de la curva de Phillips más allá del corto plazo.

Esta primera forma de racionalidad, derivada de la simple observación estadística de la serie del precio, corresponde a lo que se podría llamar racionalidad "débil"; no requiere que los agentes conozcan el modelo que rige la formación de precios y del producto en la economía. Una hipótesis adicional consiste precisamente en suponer que el público, a través de sus observaciones, experiencia y/o la labor de convencimiento de los economistas, conoce (o cree conocer) el modelo subyacente. En este caso (de racionalidad "fuerte") toma en cuenta su conocimiento del modelo para normar sus expectativas; o, en otras palabras, deriva el precio esperado directamente a partir de la ecuación verdadera de formación del precio [ecuación (17)]. Al tomar el valor esperado de esta ecuación, y dado que el valor esperado de los choques estocásticos es cero (son ruido blanco), se encuentra que:

$$
p^{\mathrm{e}}=m^{\mathrm{e}}-a\left(\gamma y^{*}+v\right)
$$

Sustituyendo este valor en la ecuación (17) se deriva que:

$$
p-p^{\mathrm{e}}=a\left(m-m^{\mathrm{e}}\right)
$$

Esta última relación comprueba lo que se expuso anteriormente: sólo los cambios no anticipados en la oferta monetaria $\left(m \neq m^{\mathrm{e}}\right)$ tienen un efecto sobre el ingreso; los cambios anticipados $\left(m=m^{\mathrm{e}}\right)$ generan expectativas correctas $\left(p=p^{e}\right)$ y repercuten en una alza de precio exactamente proporcional al aumento de la oferta monetaria. ${ }^{31}$ Este nuevo modelo se comporta, por lo tanto, exactamente como el modelo clásico tradicional cuando las acciones del gobierno son anticipadas por el público.

Finalmente, se puede notar también en este modelo que la inefectividad de la política monetaria para alterar en forma duradera el producto y el empleo va a la par con una igual incapacidad para evitar que estas variables oscilen, ya que el gobiemo tarda en conocer (o poder reaccionar a) los choques estocásticos que afectan la oferta y demanda. Además, dado que no tiene ventaja comparativa alguna respecto del público en conocer estos choques, el manejo de la oferta monetaria como instrumento de estabilización del producto se vuelve irrelevante: cuando el gobierno se encuentra en condiciones de alterar la masa

31 Esto último se deduce fácilmente de la ecuación (17) al reemplazar $p^{e}$ por $p$. 
monetaria, los agentes económicos ya incorporaron también los choques existentes dentro de sus expectativas de precios y alteraron sus ofertas y demandas de tal forma que los mercados regresarán al equilibrio. ${ }^{32}$

Las implicaciones de política están, entonces, muy cerca de las que se habian encontrado en el modelo clásico tradicional: abstenerse de intervenir y limitarse a hacer crecer moderadamente la masa monetaria. En el nuevo modelo clásico, hay que añadir además otra recomendación: que el ritmo de crecimiento de la oferta monetaria sea lo suficientemente uniforme como para evitar las "sorpresas" monetarias que desestabilizarían la actividad económica.

\section{El enfoque keynesiano}

El nuevo modelo clásico que nació como una crítica a la vieja ortodoxia keynesiana de la post-guerra ha sido a su vez recientemente el objeto de críticas crecientes. Una de estas críticas concierne las hipótesis poco realistas y un tanto arbitrarias hechas por los nuevos clásicos sobre la diseminación de la información. Ellos suponen, por una parte, que los trabajadores están lo suficientemente bien informados y son racionales como para conocer perfectamente el modelo que rige la economía y como para no tardar más tiempo que el gobierno en ajustar sus expectativas y su comportamiento cuando ocurren choques; pero por otra parte, los trabajadores tardan sistemáticamente más que las empresas en realizar estos ajustes, lo que parecería indicar que existe una barrera muy costosa para adquirir información oportuna sobre los movimientos del índice de precios. Esta asimetría parece difícil de justificar sin examinar más a fondo las características generales de una economía con información imperfecta.

Se ha argumentado en particular, en épocas recientes, que la existencia de información imperfecta tiende a crear costos de ajuste importantes en el comportamiento de las empresas y de los trabajadores. Por ejemplo, en un contexto de información imperfecta sobre las características y precios de los bienes, el cambio de precio de un producto no es conocido inmediatamente por el público en general. La empresa que reduce su precio en tiempos de escasa demanda ve mermados sus ingresos inmediatamente después del ajuste, debido a la baja en el precio y a la falta de aumento en la cantidad adquirida. Frente a este costo de ajuste, es posible entonces que sea más conveniente, en el caso de choques pequeños, mantener el precio anterior, aunque la empresa se encuentre ahora en desequilibrio. Sólo en el caso de desequilibrios más fuertes podría ser deseable realizar un ajuste de precios. ${ }^{33}$

En una economía que cuenta además con miles de productos heterogéneos, los costos de información son tales que no es posible organizar mercados centra-

32 Notese, sin embargo, que la política monetaria sigue cumpliendo un papel efectivo para estabilizar precios, en la medida en que la duración de los choques es mayor que el tiempo requerido por el gobierno para actuar.

33 Vale la pena notar que la respuesta de los precios a los cambios en la demanda tiende por lo tanto a ser altamente no lineal en el paradigma keynesiano: los precios no se alteran o se alteran muy poco cuando la demanda se mueve dentro de cierto rango alrededor del equilibrio; sufren ajustes mucho más marcados cuando el nivel de demanda rebasa estos límites. 
les que permitan igualar en todo momento ofertas y demandas para todos los productos. Los mercados se organizan en realidad alrededor de un gran número de establecimientos industriales o comerciales dispersos que tienden a fijar sus precios en función del costo promedio o "normal" de los productos, y a modular los cambios en la demanda mediante variaciones de inventarios. Estos establecimientos no ajustan sus márgenes conforme varíe el nivel de demanda agregada porque sus funciones de costos son casi horizontales y porque los costos involucrados en conocer exactamente el estado de la demanda agregada, en alterar administrativamente los precios y en difundir estos cambios entre los compradores son demasiado elevados en relación con el aumento en los ingresos que pudiera esperarse de estos cambios.

Es de notar, por otra parte, que estos costos de ajuste tienden a fijar aún más los precios si el público espera que los choques sean de duración relativamente breve. En particular, si los agentes consideran como asegurada la intervención del gobierno para estabilizar la economía, no creerán necesario y justificable reducir sus precios de venta en una época de depresión, prefiriendo esperar a que las autoridades monetarias hayan tomado por sí solas las medidas necesarias para salir rápidamente de la crisis. ${ }^{34}$

En el mercado de trabajo, la combinación de costos de ajuste y de expectativas inelásticas probablemente puede también explicar una buena parte de las rigideces observadas en los salarios. En una época de depresión, por ejemplo, las empresas evitan contratar trabajadores desempleados, aun a un nivel de salario muy bajo, porque el costo de entrenamiento del trabajador sería excesivo para las empresas comparado con el beneficio asociado con su trabajo durante una época recesiva que se supone será breve; los trabajadores, por su parte, prefieren gozar de su tiempo libre y estar listos para conseguir un buen empleo cuando termine la recesión, en vez de trabajar ahora a un nivel de sueldo muy bajo. ${ }^{35}$

Los costos administrativos inherentes en las negociaciones salariales y en los ajustes de salarios obligan, por otra parte, a las empresas a limitar la frecuencia de las revisiones salariales y a establecer contratos que fijan las remuneraciones de los trabajadores por un tiempo determinado. Pero a partir del momento en que existe cierto traslape entre las fechas de revisión de contratos para diferentes trabajadores, no se puede realizar un ajuste simultáneo de todos los salarios (y de los precios) hacia abajo en caso de que tal ajuste fuera necesario para mantener el pleno empleo (por ejemplo, porque el acervo monetario es demasiado bajo). El mantenimiento del pleno empleo requeriría, entonces, que los trabajadores cuyos contratos vencen en el período actual aceptaran reducciones salariales, ya no sólo nominales sino también reales, que compensaran el aumento producido por

34 Es interesante notar que el éxito estabilizador de las políticas keynesianas de la postguerra pudo así haber contribuido en alguna forma a hacer más rígida la estructura de precios en años recientes. Este argumento se encuentra, por ejemplo, desde la década del ciencuenta en los escritos de Morton (1950).

$35 \mathrm{El}$ hecho que la duración de la jornada de trabajo tienda por otra parte a ser inflexible ( 8 horas por día) refleja probablemente también la existencia de costos tijos importantes en la relación empresa-trabajador: administrativos y de entrenamiento para la empresa; de transporte y formación para el trabajador. 
las reducciones de precios en el sueldo real de los demás trabajadores. Esta perspectiva conduce a los que ya tienen que renegociar su sueldo a no aceptar estas reducciones y a preferir cierto desempleo junto con una reducción más moderada de su sueldo. ${ }^{36}$

Debido a todas estas rigideces, los únicos sectores en donde probablemente exista una más amplia capacidad de respuesta de los precios a los cambios de oferta y demanda, son los sectores de insumos primarios cuyos mercados son más competitivos, es decir más centralizados y mejor organizados, por ejemplo, los de bienes agrícolas, mineros o industriales básicos. Sin embargo, aun si existen mercados de este tipo, es probable que el impacto sobre el conjunto de la economía de cambios en las condiciones de oferta y demanda se haga sentir sólo con cierto atraso: por un lado, porque la existencia de inventarios retrasa la transmisión hacia los sectores primarios de cambios en la demanda final; por otro lado, porque cambios en los costos primarios tienden a reflejarse con cierto atraso en el precio de los productos elaborados.

Por todas estas razones, que como se acaba de ver, están generalmente asociadas con deficiencias generalizadas de información, existen entonces rigideces importantes en los movimientos de los precios y salarios, que no contemplan los modelos clásicos. La originalidad del paradigma keynesiano consiste, precisamente, en incorporar la hipótesis de completa rigidez dentro de sus modelos. Esta hipótesis, aunque seguramente un poco extrema, proporciona un punto de referencia alternativo muy útil para efectos comparativos. ${ }^{37}$ Se desarrollará a continuación un modelo "keynesiano" particularmente sencillo para ilustrar en forma un poco más precisa este último punto.

Se supondrá que se parte de un modelo similar al clásico pero en el cual el precio y el salario están dados y no pueden ajustarse para equilibrar los mercados. Se supondrá también, para mayor simplicidad, que la oferta de trabajo es constante y que la oferta monetaria está fija. Este último supuesto implica que el gasto público es nulo. Finalmente, en vez de examinar el desequilibrio en el mercado monetario, será más conveniente analizar el del mercado del bien, partiendo de una función de gasto privado. Estos supuestos conducen a un modelo del tipo siguiente:

$$
y^{o}=\alpha \min \left\{l^{o}, l^{D}\right\}+\beta
$$

36 La contribución básica sobre este proceso de ajuste salarial es la de Taylor (1979). Es interesante notar, que el desempleo en este modelo es consistente con la existencia de contratos eficientes, o sea de contratos en los cuales tanto la empresa como el trabajador maximizan su utilidad, dadas las restricciones institucionales existentes en el mercado laboral. Existe también una amplia literatura que intenta explicar rigideces salariales dentro de un contexto de contratos eficientes, a partir de aversiones al riesgo diferentes entre empresas y trabajadores [véase, por ejemplo Baily (1974 y 1977), Azariadis (1977) y la reseña en Hall (1980)]. Según esta literatura, puede ser óptimo para ambas partes que la empresa "asegure" al trabajador un sueldo constante. Como lo demuestra sin embargo Bliss (1981), estos modelos parten generalmente de hipótesis restrictivas bastante cuestionables.

37 En un modelo keynesiano más general, los precios y salarios podrian ser rígidos en el muy corto plazo pero se ajustarían lentamente después hacia sus valores de equilibrio. 


$$
\begin{aligned}
& l^{D}=\alpha^{\prime}(p-w)+\beta^{\prime} \\
& \rho=\bar{l} \\
& y^{D}=\gamma(m-p)+\nu \\
& y=\min \left\{y^{o}, y^{D}\right\} \\
& l=(y-\beta) / \alpha
\end{aligned}
$$

Las ecuaciones (20) y (21) expresan que la oferta de bienes es la misma del modelo clásico [véanse las ecuaciones (1) y (2) ] excepto cuando la demanda de trabajo de equilibrio, $l^{D}$, rebasa la oferta de trabajo disponible, en cuyo caso la oferta de bienes está limitada por la oferta de trabajo. La ecuación (22) define está oferta de trabajo.(23) es ahora la función de gasto del sector privado, que, en ausencia de gasto público, corresponde al gasto total. ${ }^{38}$ Finalmente, (24) indica que la cantidad del bien realmente intercambiada está dada por la menor de dos magnitudes, oferta y demanda, y (25) relaciona, a través de la función de producción, las cantidades intercambiadas del bien y del trabajo.

Dado que hay dos mercados en esta economía existen cuatro posibles combinaciones de desequilibrio. Sin embargo, en este modelo puramente estático, una de ellas, la que asocia un exceso de demanda de trabajo con un exceso de oferta del bien, no puede ocurrir puesto que la existencia de exceso de demanda de trabajo señala necesariamente que existe demanda insatisfecha del bien. Quedan por lo tanto tres combinaciones posibles.

La primera, que se traduce por exceso de oferta en ambos mercados, es la que puede denominarse como propiamente keynesiana, dado que corresponde a una depresión generalizada, con desempleo y exceso de capacidad instalada. La segunda, de exceso de oferta de trabajo y exceso de demanda del bien, ha sido llamada "clásica" porque, como se verá a continuación, está causada por un salario real demasiado alto. La tercera, de exceso de demanda en ambos mercados, podría por su parte llamarse "inflación reprimida".

Las fronteras respectivas de estas diferentes combinaciones o regiones pueden ubicarse fácilmente dentro de un plano salario real/saldo monetario real. La frontera entre las regiones clásicas y keynesianas se caracteriza por el hecho de que el mercado del bien está en equilibrio sobre la frontera, mientras que el de trabajo debe estar en desequilibrio por exceso de oferta. ${ }^{39}$

Esto implica que:

$$
y^{D}=\gamma(m-p)+\nu=y^{o}=\alpha l^{D}+\beta
$$

38 En un modelo más riguroso, esta función de gasto debería alterarse conforme la economía se encuentra en una u otra región de desequilibrio [véase Malinvaud (1977)]. Aquí, para mayor simplicidad, se supondrá invariable en todo el espacio.

39 Existe exceso de demanda del bien, del lado clásico, exceso de oferta del lado keynesiano, y de ambos lados exceso de oferta de trabajo. Es claro, por lo tanto que, en la frontera, el mercado del bien debe estar en equilibrio y el de trabajo en desequilibrio por exceso de oferta. 
La ecuación de la frontera es entonces

$$
\gamma(m-p)+\nu=\alpha \alpha^{\prime}(p-w)+\alpha \beta^{\prime}+\beta
$$

La frontera entre las regiones clásicas y de inflación reprimida se caracteriza, por su parte, por el hecho de que el mercado de trabajo tiene que estar en equilibrio y el del bien en desequilibrio por exceso de demanda ${ }^{40}$ Esto implica que:

$$
l^{D}=\alpha^{\prime}(p-w)+\beta^{\prime}=l^{O}=\bar{l}
$$

La ecuación de la frontera es entonces:

$$
\alpha^{\prime}(p-w)+\beta^{\prime}=\bar{l}
$$

Finalmente, en la frontera de las regiones keynesianas y de inflación reprimida, debe existir pleno empleo y un mercado del bien en equilibrio, ${ }^{41}$ por lo que:

$$
y^{D}=\gamma(m-p)+\nu=y^{o}=\alpha \bar{l}+\beta
$$

y la ecuación de la frontera es:

$$
\gamma(m-p)+\nu=\alpha \bar{l}+\beta
$$

El mapa de estas regiones se deduce inmediatamente de las ecuaciones (27), (29) y (31): es fácil comprobar que estas tres rectas se intersectan en un punto común en donde ?mbos mercados están en equilibrio (nocional); éste puede definirse como el equilibrio general walrasiano, W. Como se aprecia en la gráfica 1, la región keynesiana es la del bajo saldo monetario, la clásica es la del sueldo real alto y la de inflación reprimida, la de bajo sueldo real y alto saldo monetario. Para alcanzar el equilibrio general, hace falta, en general, ajustar tanto el saldo monetario como el salario real.

La determinación del producto en cada una de las tres regiones puede obtenerse simplemente a partir de las ecuaciones del modelo. En la región keynesiana es:

$$
y=\gamma(m-p)+\nu
$$

en la clásica:

$$
y=\alpha \alpha^{\prime}(p-w)+\alpha \beta^{\prime}+\beta
$$

40 El razonamiento para deducir esta afirmación es similar al del caso anterior.

41 Es de notarse, sin embargo, que en este caso (y excepto cuando el salario real tiene su valor de equilibrio general), los mercados de trabajo y del bien están en equilibrio "efectivo", y no "nocional", según la terminología de Clower: la demanda de trabajo cuando las empresas no se encuentran restringidas en el mercado del bien, que, por definición, es ${ }^{D}$ no es necesariamente igual a la oferta $l O$. Por otra parte, la oferta "nocional" del bien, $\alpha l^{D}$ $+\beta$, es también mayor a la demanda. En esta frontera, el nivel "efectivo" de oferta del bien, $\alpha \bar{l}+\beta$, coincide con el nivel de demanda, y el nivel "efectivo" de demanda de trabajo coincide con la oferta, $\bar{l}$. 


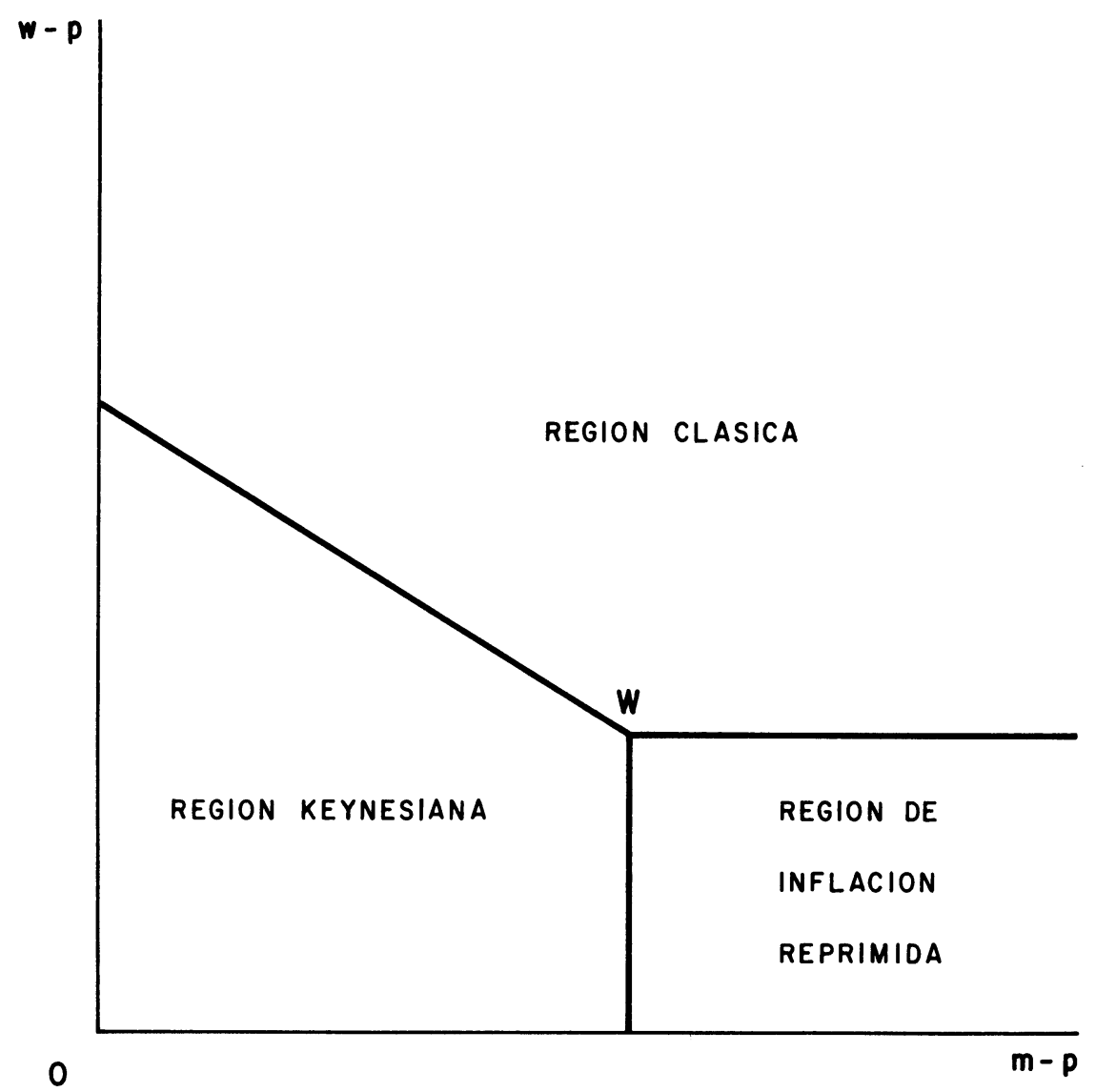

Gráfica 1. Regiones de desequilibrio

y en la de inflación reprimida:

$$
y=\alpha \bar{l}+\beta
$$

Se aprecia inmediatamente, a partir de estas ecuaciones, que el gobierno puede elevar el producto en las dos primeras regiones; mediante estímulos monetarios en la región keynesiana, reducciones en el salario real en la clásica. Esto último ilustra bien el debate entre Keynes y los clásicos durante la gran depresión: mientras los clásicos argumentaban que se requería una reducción del salario real para salir de la crisis, Keynes proponía estímulos fiscales y monetarios. El éxito de las recomendaciones de Keynes obedece, según este modelo, a que la economía mundial se ubicaba entonces en una región de exceso de capacidad disponible.

Las conclusiones generales del análisis keynesiano son entonces, por una parte, que el gobierno puede cumplir un papel activo y útil como regulador del nivel de 
actividad. económica, mediante el manejo de la política fiscal y monetaria; y, por otra parte, que la relación entre dinero y precios no es una relación necesariamente tan inmediata y sencilla como lo sugerían las teorías clásicas. La economía podría estar en una región en la cual una variación moderada en la cantidad de dinero no tendría prácticamente efecto alguno sobre el nivel de precios; sólo cambios mayores alcanzarían a causar alteraciones de demanda suficientemente fuertes como para provocar ajustes de precios importantes.

\section{Los enfoques sociológicos}

La hipótesis central que los enfoques sociológicos tratan de descartar ya no es tanto la de información perfecta, como en el caso de los nuevos clásicos y de los keynesianos, sino más bien la de perfecta competencia. ${ }^{42}$ Se trata ahora de analizar los movimientos del índice de precios y del producto cuando los precios relativos no están determinados por el juego de la oferta y demanda en mercados perfectamente competitivos, sino que están fijados directamente por los agentes económicos, individualmente o en coaliciones.

Una de las características esenciales de los sistemas de equilibrio no competitivo parece ser la posible existencia de equilibrios múltiples, tanto en precios relativos como en cantidades. La distribución del ingreso no es por lo tanto única y la intensidad en el uso de los factores puede también variar conforme se alcanze uno u otro equilibrio. El proceso de convergencia hacia un posible equilibrio depende fundamentalmente del poder de negociación de cada agente o grupo social y de sus expectativas sobre el comportamiento de los demás agentes, ya que cada una de sus acciones provocarán una reacción de otros individuos o grupos que tratan de defender sus intereses económicos. Este tipo de dinámica quizás permita explicar cierta rigidización de la estructura de precios relativos que parece darse en la práctica y que forma el común denominador de estos enfoques. ${ }^{43}$

Un modelo muy sencillo que incorpore esta característica podría ser el que contemple solamente dos grupos sociales, empresas y trabajadores. Las empresas fijan su margen de ganancia, los trabajadores su sueldo real esperado. De tal forma que

$$
y=\alpha l+\beta, \alpha<1
$$

42 Es necesario recalcar, sin embargo, que ambas hipótesis están, hasta cierto punto, interconectadas; es difícil, por ejemplo concebir una economía con competencia perfecta en un mundo de información imperfecta. La diferencia está aquí en el énfasis sobre uno u otro aspecto.

43 La literatura sobre equilibrios no competitivos está aún relativamente poco desarro llada, aunque crezca rápidamente; véase en particular Negishi (1960) y (1979), Hahn (1977a y b) y (1978), Nikaido (1975) y la reseña en Schotter y Schwodiauer (1980). Otras contribuciones han empezado a surgir desde un enfoque más directo de teoría de juegos. Véase por ejemplo Marschak y Selten (1974 y 1978) y la reseña en Schotter y Schwodiauer (1980). Un aporte reciente de particular interés es el de McDonald y Solow (1981).

44 Para un análisis empírico reciente de estos aspectos, véase en particular los estudios históricos sobre tasas de ganancia y salarios reales de Sachs (1979), Boyer (1979) y Benassy, Boyer y Gelpi (1979). 


$$
\begin{aligned}
& p+y=l+w+a \\
& w=p^{e}+b \\
& m^{D}=p+\gamma y+\nu \\
& m^{D}=m
\end{aligned}
$$

(35) es la función de producción; (36) es la regla de margen constante de ganancia; (37) el sueldo real esperado. (38) y (39) son las condiciones usuales de demanda de dinero y de equilibrio en ese mercado. La cantidad de trabajo contratada está fijada por el lado de la demanda y existe generalmente desempleo.

La resolución del modelo es inmediata. De las ecuaciones (35), (36) y (37) se deriva que

$$
p-p^{e}=a+b-\beta+(1-\alpha) l
$$

Supóngase primero que $\alpha$ sea estrictamente menor que uno (existen retornos decrecientes en el uso del trabajo). La condición (40) expresa entonces que el equilibrio en expectativas $\left(p=p^{e}\right)$ requiere que el nivel de empleo $(l)$ se ajuste para armonizar las peticiones de los agentes $(a$ y $b)$ con el nivel de productividad $(\alpha$ y $\beta)$. Un incremento en estas peticiones no acompañado por un alza en productividad forzará así el nivel de empleo hacia la baja, de tal forma que el aumento correspondiente en la productividad marginal del trabajo permita compensar el incremento inicial en las peticiones. Se nota, sin embargo, al revisar el modelo keynesiano que este resultado es el que ya había sido obtenido en la región "clásica" [véase la ecuación (33) ] en donde una alza del sueldo real restringía producción y empleo. ${ }^{45}$ El problema que enfrenta la economía y su solución es el mismo en ambos casos: un exceso de peticiones que repercute en una reducción del empleo.

Pero supóngase ahora que existe poca capacidad de sustitución en el corto plazo entre capital y trabajo, y que la productividad marginal del trabajo es constante (dentro de un margen amplio de utilización de la capacidad instalada), de tal forma que la función de producción sea lineal: $\alpha=1$. Se aprecia entonces, a partir de la ecuación (40), que ajustes en el empleo ya no permiten reconciliar las diferencias que puedan surgir entre peticiones y productividad; estas diferencias tienen entonces que verse reflejadas necesariamente en discrepancias entre precios reales y precios esperados. En la medida en que los agentes económicos son racionales, va a darse un proceso inflacionario explosivo mientras siga existiendo algún conflicto entre peticiones y productividad $(a+b \neq \beta)$. Como se vio anteriormente, existen varias maneras, dentro de los enfoques sociológicos, de explicar estas divergencias, desde choques estocásticos de oferta hasta tendencias históri-

45 Se aprecia de hecho, que la estructura de ambos modelos es idéntica en este caso: la regla de margen constante de ganancia viene a reemplazar la ecuación de demanda competitiva por trabajo pero el resultado algebraico obtenido en combinación con la función de producción tiene la misma forma. 
cas de acumulación. La mecánica de la inflación es, sin embargo, la misma en todos los casos.

Del lado del producto, se observa que aquí como en el caso keynesiano, cambios en la cantidad de dinero permiten alterar el nivel del producto:

$$
y=(m-p) / \gamma-v / \gamma
$$

Pero se aprecia también que un alza de precios causada por un conflicto entre peticiones y productividad tenderá a hacer caer el producto a menos que el gobierno lo estabilice mediante un incremento en la oferta monetaria. En particular, si el gobierno intenta estabilizar el producto alrededor de un nivel de pleno empleo, $y^{*}$, la oferta monetaria se vuelve endógena:

$$
m=p+\gamma y^{*}+\nu
$$

Al "financiar" entonces sistemáticamente las alzas de precios causadas por conflictos sociales, el dinero sigue a los precios y no los precios al dinero, como en el caso de los clásicos. La relación dinero/precios sigue por lo tanto existiendo pero la dirección de causalidad se ha invertido radicalmente. Esta observación concentra una gran parte del debate entre las escuelas monetaristas y estructuralistas.

\section{Modelos ALternativos EN UNA ECONOMÍA ABIERTA}

\section{El enfoque neoclásico}

Todo lo expuesto hasta ahora ha permanecido dentro del marco de una economía cerrada. Resulta ahora necesario ampliar este marco de análisis al caso de economías abiertas. Tal como se hizo anteriormente para las economías cerradas, se propondrán tres tipos de enfoques distintos, el neoclásico, el keynesiano y el sociológico, y se analizarán las propiedades básicas de estos tres enfoques con la ayuda de un modelo cuyas características esenciales sean comunes.

El modelo desarrollado aquí tiene dos sectores productivos (uno de bienes comerciables y otro de no comerciables) y sigue teniendo un solo insumo, trabajo. Se seguirá también considerando una economía con un solo activo financiero (dinero), excluyéndose por lo tanto lo referente a movimientos de capitales privados en balanza de pagos. La versión neoclásica de este modelo será la que sigue: ${ }^{46}$

$$
\begin{aligned}
& Y_{N}=f_{N}\left(L_{N}^{D}\right), f_{{ }_{N}}^{\prime}>Q, f_{N}^{\prime \prime}<0 \\
& Y_{T}^{O}=f_{T}\left(L_{T}^{D}\right), f_{T}^{\prime}<0, f_{T}^{\prime \prime}<0
\end{aligned}
$$

46 Debido a la existencia de dos sectores, resultaría incómodo usar un formato logarítmico, por lo que el modelo está expresado en un formato funcional más general y las variables están en sus magnitudes naturales. 


$$
\begin{aligned}
& L_{N}^{D}=g_{N}\left(P_{N} / W\right), g_{N}^{\prime}>0 \\
& L_{T}^{D}=g_{T}\left(e P_{T} / W\right), g_{T}^{\prime}>0 \\
& L^{0}=h(W / P), h^{\prime}>0 \\
& P=P_{N}^{\alpha}\left(e P_{T}\right)^{1-\alpha} \\
& L^{O}=L_{N}^{D}+L_{T}^{D} \\
& Y_{N} / Y_{T}^{D}=\frac{\alpha}{1-\alpha} \frac{e P_{T}}{P_{N}} \\
& M^{D}=k\left(P_{N} Y_{N}+e P_{T} Y_{T}^{O}\right) \\
& M^{D}=M \\
& P_{T}\left(Y_{T}^{D}-Y_{T}^{0}\right)=Z
\end{aligned}
$$

(43) y (44) son las funciones de producción (neoclásicas) de los sectores de bienes no comerciables $(\mathrm{N})$ y comerciables $(T) .{ }^{47}(45)$ y $(46)$ son las demandas de trabajo que se derivan de estas funciones en un contexto competitivo. El precio (en moneda extranjera) de los comerciables, $P_{T}$, está fijado internacionalmente; $e$ es la tasa de cambio. (47) es la oferta de trabajo y (48) el índice de precios. ${ }^{48}$ (49) es la condición de equilibrio en el mercado de trabajo. (50) refleja las elasticidades-precio de las demandas de comerciables y no comerciables. (51) es la función de demanda de dinero usual. Finalmente (52) y (53) corresponden a la condición de equilibrio en el mercado de dinero y a la ecuación de balanza de pagos, $\mathrm{Z}$ siendo el déficit en cuenta corriente, que es financiado por incrementos en la deuda externa (o, como en el caso de México, por exportaciones de petróleo).

La solución de este modelo es bastante sencilla: el primer paso consiste en ver que el bloque de oferta (ecuaciones (43) a (49)) permite obtener la oferta de comerciables y de no comerciables en función del índice de precios relativos definido como:

$$
X=e P_{T} / P_{N}
$$

En efecto, si se usan las ecuaciones de demanda y oferta de trabajo y si se expresa el índice de precios en función de $\mathrm{Z}$, la condición de equilibrio en el mercado de trabajo puede ser escrita como:

47 Dados los ajustes que pueden darse a través de la cuenta corriente, la oferta y demanda por comerciables no son necesariamente iguales y es, por lo tanto, necesario identificar en forma separada ambos componentes, $Y_{T}^{O}$ y $Y_{T}^{D}$.

48 Se está suponiendo aquí que el sistema de gasto es lineal. 


$$
g_{N}\left(P_{N} / W\right)+g_{T}\left(X P_{N} / W\right)=h\left(X^{\alpha-1} W / P_{N}\right)
$$

Dado los signos de las derivadas de $g_{N}, g_{T}$ y $h$, es claro que la ecuación (55) permite obtener la relación $P_{N} / W$ como una función inversa de $\mathrm{X}$; de ahí se deriva inmediatamente con (43) y (45) que $Y_{N}$ es una función inversa de $X$; y de la misma forma, se obtendría que $Y_{T}{ }_{T}$ es una función creciente de esta variable. Las ofertas de comerciables y no comerciables están por lo tanto sobre una frontera de producción con pendiente negativa sobre el espacio $\left(Y_{N}, Y_{T}^{O}\right)$. Dado que las empresas están maximizando ganancias a todo lo largo de esta frontera, la pendiente en cada punto (la tasa marginal de transformación) debe además ser igual a la relación de precios, $X$ Esio asegura, por lo tanto, concavidad de la frontera.

Por otra parte, al expresar $Y_{N}$ y $Y_{T}^{0}$ como funciones de $X$ en la ecuación de demanda de dinero y tomando en cuenta la condición de equilibrio monetario y la igualdad entre la tasa marginal de transformación y la relación de precios a lo largo de la frontera de producción, es fácil deducir que $X$ es una función inversa del saldo monetario real expresado en términos del precio de los comerciables: ${ }^{49}$

$$
X=X\left(M / e P_{T}\right), X^{\prime}<O
$$

De esta relación, se deduce inmediatamente, usando la definición de $X$ en (54) y de $P$ en (48), que:

$$
P=e P_{T} F\left(M / e P_{T}\right), F^{\prime}>O
$$

Por otra parte, usando la ecuación (50) y las expresiones de $Y_{N}$ y $Y_{T}^{0}$ como funciones de $X$ en la ecuación de balanza de pagos se deduce inmediatamente que el déficit comercial (en términos reales) es una función inversa de $X$; de ahí, con (56), que: ${ }^{50}$

$$
Z / P_{T}=G\left(M / \text { e } P_{T}\right), G^{\prime}>O
$$

49 La ecuación de demanda de dinero se escribe

$$
\frac{M}{e P_{T}}=k\left[Y_{N} / X+Y_{T}^{D}\right]
$$

$$
\text { de ahí que: } \quad \frac{d(M)}{e P_{T}}=k\left[\frac{d y_{N}}{X}+d y_{T}^{O}-Y_{N} \frac{d x}{X^{2}}\right]
$$

$$
\text { Pero dado que: } \frac{d y_{N}}{d y_{T}^{O}}=-X \text {, resulta que } d X=\frac{-X^{2}}{y_{N}} d\left(\frac{\mathrm{M}}{e}\right)
$$

50 Se observa así que el déficit en cuenta corriente es exclusivamente función del saldo monetario. Esto constituye la esencia del enfoque "monetario" de la balanza de pagos [sobre este enfoque, véase en particular Mundell (1968) y Frenkel y Johnson (1976)]. 
Finalmente, se sabe, dado que $Y_{N}$ y $Y_{T}^{0}$ son funciones de $X$ y que $X$ es a su vez una función del saldo monetario real, que:

$$
\begin{aligned}
& Y_{N}=Y_{N}\left(M / e P_{T}\right), Y_{N}^{\prime}>0 \\
& Y_{T}=Y_{T}\left(M / e P_{T}\right), Y_{T}^{\prime}<0
\end{aligned}
$$

El conjunto de ecuaciones (57) a (60) permite sintetizar el comportamiento del modelo. Se aprecia que el gobiemo puede hacer uso de tres instrumentos de control: la oferta monetaria, $M$, la tasa de cambio, $e$, y el endeudamiento, $Z$.

Existen sin embargo, dos ecuaciones que restringen el uso de estos instrumentos; por una parte, la ecuación de balanza de pagos [ecuación (58)], y, por otra parte, la restricción presupuestaria del gobierno, $G=M+e Z$. Si se supone, sin embargo, que el gobierno puede ajustar libremente $G^{51}$ sigue disponiendo de dos instrumentos independientes dentro del conjunto $(Z, e, M)$.

Si se considera en particular una política que mantenga constante el déficit en cuenta corriente, se observa que la tasa de cambio debe seguir los movimientos de la oferta monetaria, de tal forma que $M / e P_{T}$ permanezca constante. Al revisar las ecuaciones (57), (59) y (60) se concluye que esta política permite que las ofertas de bienes permanezcan inalteradas y que los precios crezcan al mismo ritmo que la oferta monetaria. Se vuelve por lo tanto a encontrar el mismo resultado que en el caso de una economía cerrada: la inflación es un fenómeno puramente monetario y el dinero es neutral en cuanto a su impacto real. La tasa de cambio flotante permite así "aislar" la economía y hacerla funcionar como si estuviera cerrada. ${ }^{52}$

Si se considera por otra parte una política que mantenga constante el déficit en cuenta corriente, pero que mantenga al mismo tiempo fija la tasa de cambio, se deduce inmediatamente de la ecuación (58) que la oferta monetaria debe crecer al mismo ritmo que la inflación externa, y que [véase a la ecuación (57)] la inflación interna sigue exactamente a la externa. Las autoridades monetarias pierden entonces el control independiente de los precios y dejan a la economía en una posición de apertura total hacia las perturbaciones externas.

Finalmente, si el gobierno deja ensancharse el déficit en cuenta corriente, por ejemplo mediante aumentos en la oferta monetaria que rebasan los ritmos combinados de devaluación y de inflación externa, se observa que el nivel interno de precios sube más rápidamente que el extemo, se deforma la estructura de precios relativos ( $X$ se reduce) y aumenta la producción de no comerciables mientras que se restringe la de comerciables. El impacto del dinero cesa de ser neutral y la inflación se vuelve entonces el resultado combinado de la inflación externa, de la oferta monetaria y de las modificaciones en la tasa de cambio.

51 Esto implica que el gobierno puede "esterilizar" sistemáticamente los cambios indeseados en la oferta monetaria provenientes de movimientos en la balanza de pagos.

52 Es necesario, sin embargo, recalcar que una tasa flotante ya no es suficiente para aislar la economía cuando existe un libre movimiento de capitales internacionales. 


\section{El enfoque keynesiano}

El modelo neoclásico que acaba de ser presentado puede ser ahora adaptado al caso keynesiano en el cual los precios y los salarios son rígidos. Sin embargo, en el contexto de una economía abierta, surgen algunos problemas nuevos.

En primer lugar, es necesario precisar si el sector de bienes comerciables se encuentra en equilibrio (i.e. si puede vender todo lo que desee en el mercado mundial, al precio $P_{T}$ ) o si se encuentra en desequilibrio por exceso de oferta (el precio de los comerciables es rígido y la oferta mundial se encuentra racionada por un nivel insuficiente de demanda efectiva). Para mayor simplicidad, y dado que esto no altera sustancialmente el modelo, se supondrá que está en equilibrio.

El segundo problema consiste en redefinir el supuesto de rigidez en los salarios y los precios, puesto que uno de los precios, el de los comerciables, ya no es rígido sino que es fijado internacionalmente y su valor en moneda nacional depende de la tasa de cambio. Una hipótesis de completa rigide ${ }^{53}$ del salario y del precio de los no comerciables implicaría que la estructura de precios relativos pudiera deformarse libremente al alterarse el precio de los comerciables. Una buena parte de los argumentos expuestos anteriormente para justificar rigideces de precios, no permitiría sin embargo justificar estas distorsiones. Parece, por lo tanto, más razonable suponer que toda la estructura de precios tiende a ajustarse en el corto plazo aunque en forma imperfecta, conforme varía el precio de los comerciables. $^{54}$

Finalmente, para mayor simplicidad, se limitará el análisis al caso estrictamente keynesiano, en la práctica quizás el más relevante, en que los mercados de bienes no comerciables y de trabajo se encuentran ambos en desequilibrio por exceso de oferta. Tomando todas estas aclaraciones en cuenta, un modelo keynesiano sencillo pero representativo podría ser el que sigue:

$$
\begin{aligned}
& Y_{N}=f_{N}\left(L_{N}^{D}\right), f_{N}^{\prime}>0, f_{N}^{\prime \prime}<0 \\
& Y_{T}^{0}=f_{T}\left(L_{T}^{D}\right), f_{T}^{\prime}>0, f_{F}^{\prime \prime}<0 \\
& L_{T}^{D}=g_{T}\left(e P_{T} / W\right), g_{T}>0 \\
& P_{N}=a\left(e P_{T}\right)^{\beta}, 0<\beta^{\prime}<1 \\
& W=b\left(e P_{T}\right) \beta, 0<\beta<l
\end{aligned}
$$

53 Nótese, sin embargo, que esta hipótesis de rigidez no significa que los precios sean absolutamente rígidos; basta con que lo sean en relación con las expectativas existentes sobre precios futuros. Los precios pueden así alterarse a través del tiempo, si esto es lo que se espera.

54 Un ajuste imperfecto del salario y del precio de los no comerciables sobre el precio de los comerciables es seguramente también lo que cualquier gobierno desearía después de una devaluación y que buscará imponer mediante controles de precios y salarios. 


$$
\begin{aligned}
& P=P_{N}^{\alpha} e P_{T}^{1-\alpha} \\
& Y_{N} / Y_{T}^{D}=\frac{\alpha}{1-\alpha} \frac{e P_{T}}{P_{N}} \\
& M^{D}=k\left(P_{N} Y_{N}+e P_{T} Y_{T}^{O}\right) \\
& M^{D}=M \\
& P_{T}\left(Y_{T}^{D}-Y_{T}^{0}\right)=Z
\end{aligned}
$$

Las ecuaciones (61) a (63) corresponden a las funciones de producción usuales y a la demanda de trabajo en el sector de comerciables. (64) y (65) indican que tanto el precio de los no comerciables como el salario se ajustan parcialmente (o totalmente, en el caso extremo en el cual los coeficientes $\beta$ y $\beta^{\prime}$ son iguales a la unidad) a los cambios en el precio (en moneda nacional) de los comerciables. Las ecuaciones (67) a (70) son las mismas que en el modelo neoclásico.

La resolución del modelo es inmediata. De las ecuaciones (64) y (66) se deduce que el índice de precios es una función de los precios externos:

$$
P=a^{\alpha}\left(e P_{T}\right)^{1-\alpha(1-\beta)}
$$

Excepto cuando el precio de los no comerciables sigue totalmente el de los comerciables $(\beta=1)$, existe sin embargo cierta inercia en esta relación.

De las ecuaciones (62), (63) y (65) se deduce por otra parte que:

$$
Y_{T}^{0}=Y_{T}\left(e P_{T}\right)^{1-\beta^{\prime}}, Y_{T}^{\prime}>0
$$

A partir de la ecuación de demanda de dinero, se concluye también que:

$$
\begin{aligned}
& Y_{N}=Y_{N}\left[M / e P_{T},\left(e P_{T}\right)^{1-\beta},\left(e P_{T}\right)^{1-\beta^{\prime}}\right],\left(Y_{N}^{\prime}\right)_{1}>0 \\
& \left(Y_{N}^{\prime}\right)_{2}>0,\left(Y_{N}^{\prime}\right)_{3}<0
\end{aligned}
$$

$\mathrm{Y}$, finalmente, expresando $Y_{T}^{D}$ como una función de $Y_{N}$ en (67) y usando las expresiones encontradas para $Y_{T}^{0}$ y $Y_{N}$ en la ecuación de balanza de pagos, se deduce que el déficit en cuenta corriente es una función de la forma siguiente:

$$
Z / P_{T}=G\left(M / e P_{T},\left(e P_{t}\right)^{1-\beta^{\prime}}\right), G_{1}>0, G_{2}<0
$$

Este sistema puede ser analizado más claramente con la ayuda de una gráfica en el espacio $\left(M / e P_{T}, e . P_{T}\right)$. A partir de las ecuaciones (68) y (71) se deduce que el ingreso real es proporcional al saldo monetario real, y por lo tanto es de la forma:

$$
Y=\frac{1}{a^{\alpha} k}\left(\frac{M}{e P_{T}}\right)\left(e P_{T}\right)^{\alpha(1-\beta)}
$$


Excepto en el caso de una completa indexación del precio de los no comerciables sobre el de los comerciables $(\beta=1)$, esta expresión indica entonces que el conjunto de puntos que corresponden a un nivel deseado de ingreso es una curva de pendiente negativa en el espacio $\left(M / e P_{T}, e . P_{T}\right)$ representada como $Y Y$ en la gráfica 2. La ecuación (74) indica, por su parte que, excepto cuando el salario está totalmente indexado sobre el precio de los comerciables $\left(\beta^{\prime}=1\right)$, el conjunto de puntos con un nivel deseado de déficit externo es una curva de pendiente positiva en ese mismo espacio ( $Z Z$ en la gráfica 2 ). Conforme la economía se desplaza hacia la derecha y hacia arriba aumenta el ingreso, mientras que conforme se desplaza hacia la izquierda y hacia arriba se reduce el déficit en cuenta corriente. El mapa se divide, por lo tanto, en cuatro regiones con diferentes combinaciones de desequilibrios internos y externos.

Se aprecia a partir de esta gráfica y de la ecuación de precios (71) que una estimulación monetaria permite impulsar el ingreso sin elevar los precios, pero eleva simultáneamente el déficit en cuenta corriente; $y$, por otra parte, que una devaluación (con $M / e P_{T}$ constante) reduce el déficit externo y eleva simultá-

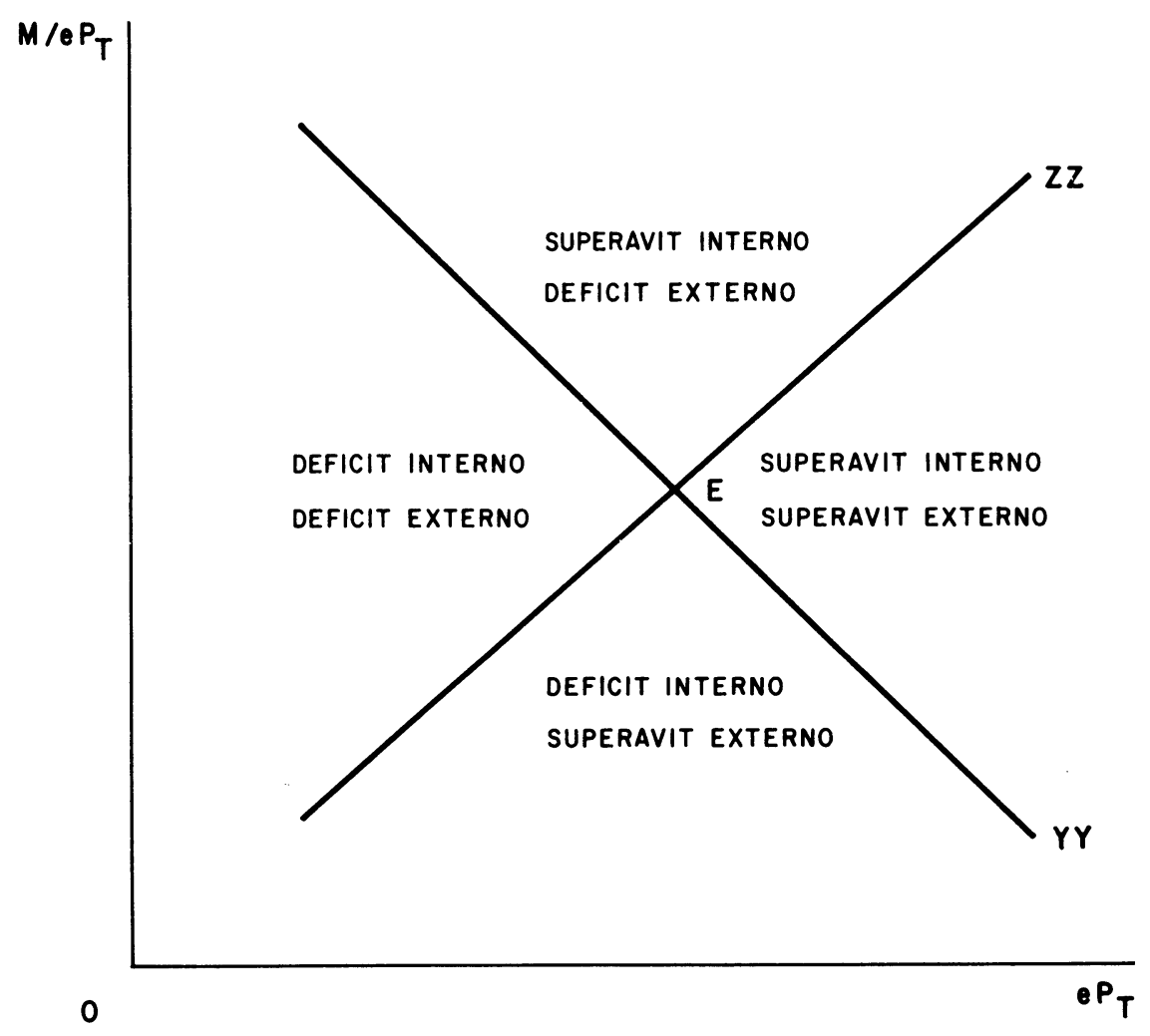

Gráfica 2. El esquema keynesiano 
neamente el ritmo de la actividad económica interna. El manejo simultáneo de la oferta monetaria y de la tasa de cambio permite así asegurar la permanencia de la economía en el punto $\mathrm{E}$ de equilibrio tanto interno como externo. Es necesario sin embargo enfatizar, para que esto sea posible, que los precios internos o los salarios no deben ajustarse totalmente sobre el precio de los comerciables después de una devaluación, de tal forma que la oferta de comerciables pueda ser estimulada y su demanda frenada. Estos son resultados típicamente keynesianos.

\section{El enfoque sociológico}

Pasando finalmente al enfoque sociológico, se hará la hipótesis de que el sector de comerciables sigue siendo perfectamente competitivo y estando en equilibrio mientras que el de no comerciables sigue una regla de determinación del precio a partir de un margen constante de ganancia y de una función de producción lineal. ${ }^{55}$ Los trabajadores, por su parte, exigen un sueldo real dado. El modelo es el que sigue:

$$
\begin{aligned}
& Y_{N}^{0}=k L_{N}^{D} \\
& Y_{T}^{0}=f_{T}\left(L_{T}^{D}\right), f_{T}^{\prime}>0, f_{T}^{\prime \prime}<0 \\
& L_{T}^{D}=g_{T}\left(e P_{T} / W\right), g_{T}^{\prime}>0 \\
& P_{N} Y_{N}^{0}=v W L_{N}^{D} \\
& W=\mu P^{e} \\
& P=P_{N}^{\alpha}\left(e P_{T}\right) 1-\alpha \\
& Y_{N} / Y_{T}^{D}=\frac{\alpha}{1-\alpha} \frac{e P_{T}}{P_{N}} \\
& M^{D}=k\left(P_{N} Y_{N}+e P_{T} Y_{T}^{0}\right) \\
& M^{D}=M \\
& P_{T}\left(Y_{T}^{D}-Y_{T}^{0}\right)=Z
\end{aligned}
$$

(76) y (79), respectivamente, son la función lineal de producción y la regla de margen constante de ganancia para el sector de no comerciables. (80) determina el sueldo a partir del precio esperado, $\mathrm{P}^{e}$. Las demás ecuaciones son las mismas que en los modelos anteriores.

Una simple eliminación algebraica entre las ecuaciones (76), (79), (80) y (81) permite obtener las relaciones siguientes:

55 El supuesto de linealidad se hace para simplificar la exposición pero no es necesario. Una función de producción cóncava daría resultados similares. 


$$
P / P^{e}=\rho X^{1-\alpha}
$$

en donde: $\rho=v \mu / k$

$$
\mathrm{y}: X=e P_{T} / P_{N}
$$

De tal forma que el equilibrio en precios $\left(P=P^{e}\right)$ requiere

$$
X=\rho^{\frac{1}{\alpha-1}}
$$

Usando por otra parte las ecuaciones (76) a (79), se deriva que:

$$
Y_{T}^{0}=Y_{T}(X), Y_{T}^{\prime}>0
$$

Finalmente, del conjunto (82) a (85), se deduce, como en los modelos anteriores, que:

$$
\begin{aligned}
& Y_{N}=Y_{N}\left(M / e P_{T}, X\right),\left(Y_{N}^{\prime}\right)_{1}>0,\left(Y_{N}^{\prime}\right)_{2} \gtrless 0 \\
& Z / P_{T}=G\left(M / e P_{T}, X\right), G_{1}^{\prime}>0, G_{2}^{\prime}<0
\end{aligned}
$$

El comportamiento del modelo puede ser visualizado satisfactoriamente con la ayuda de una gráfica en el mapa $\left(M / e P_{T}, \rho\right)$. Supóngase que el gobierno busca mantener el equilibrio en precios, de tal forma que la ecuación (89) se satisfaga. Sustituyendo $X$ por su expresión en términos de $\rho$ (derivada de (89)) en las ecuaciones (81) y (83), se concluye que el ingreso real es una función del tipo siguiente:

$$
Y=\frac{1}{k} \frac{M}{e P_{T}} \rho^{\frac{-\alpha}{1-\alpha}}
$$

El conjunto de puntos que corresponde a un nivel deseado de ingreso es por lo tanto una curva de pendiente positiva en el espacio $\left(M / e P_{T}, \rho\right)$, representada como $Y Y$ en la gráfica 3. De la misma manera, se concluye a partir de (92) que el conjunto de puntos que corresponde a un nivel deseado de déficit en cuenta corriente es una curva de pendiente positiva, representada como $\mathrm{ZZ}$ en la gráfica 3. Conforme la economía se desploma hacia abajo y hacia la derecha se reduce el nivel de actividad económica, mientras que conforme se desplaza hacia la derecha y hacia arriba aumenta el déficit externo. El mapa se divide, por lo tanto, aquí también en cuatro regiones con diferentes combinaciones de desequilibrios internos y externos.

Supóngase que la economía está inicialmente en el punto de equilibrio deseado $(E)$ y que algún choque, ya sea en la productividad de la economía o en las peticiones de los agentes, haga elevarse el parámetro $\rho$ desde su valor de equili- 


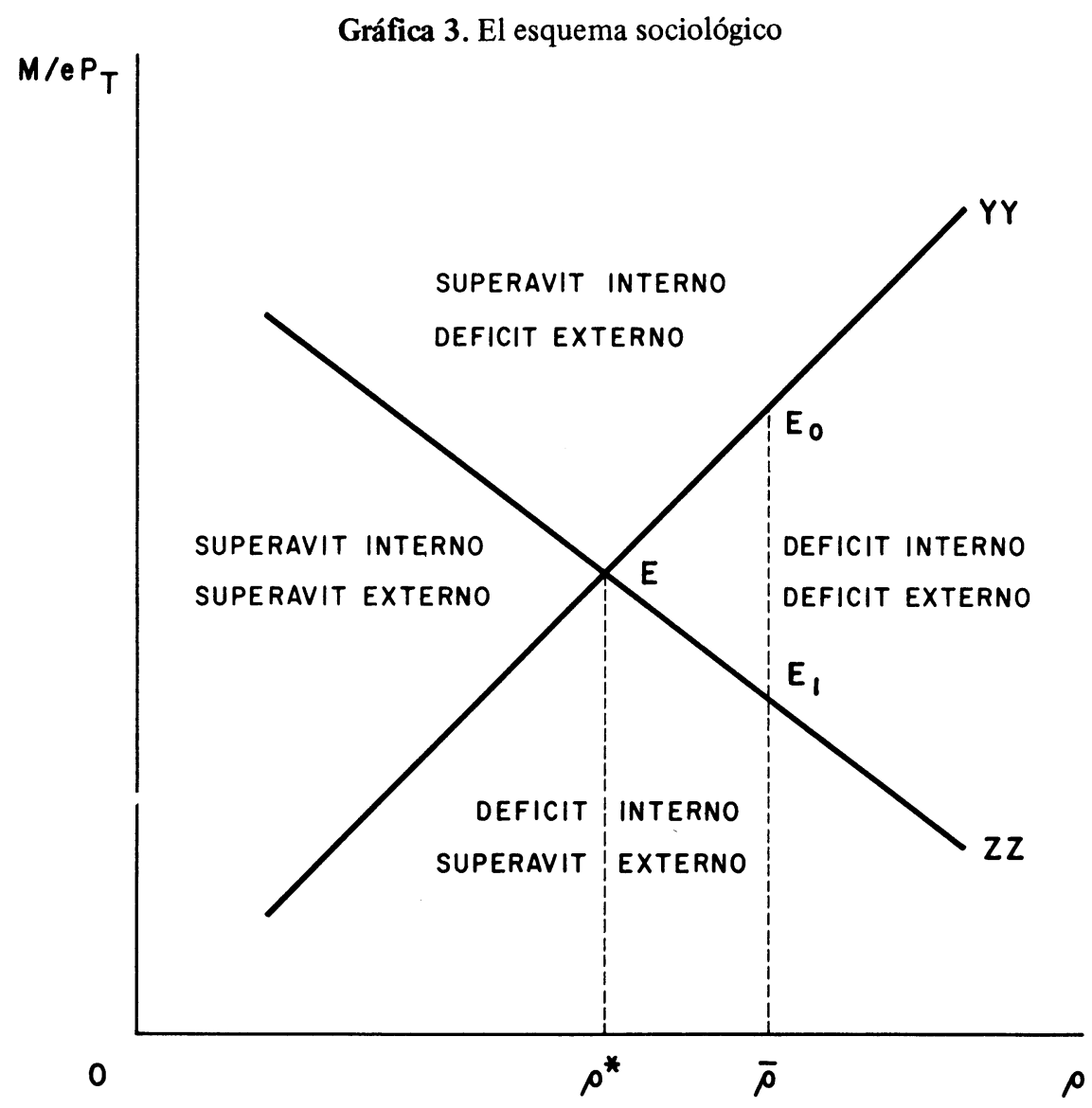

brio, $\rho^{*}$, hasta un nuevo valor, $\bar{\rho}$. El gobierno puede alterar la oferta monetaria o la tasa de cambio para tratar de restablecer el equilibrio. Pero, en este modelo, estos dos instrumentos se reducen a uno solo, ya que el único instrumento que tendrá un impacto real sobre la economía es el saldo monetario real, $M / e P_{T}$. Si el gobierno decidiera aumentar este saldo (siguiendo así una política acomodaticia que consiste en financiar los aumentos de precios causados por la elevación de $\rho,{ }^{56}$ podría mantener la economía en el punto $E_{O}$ de la curva $E E$ de empleo deseado: el costo asociado con esta política sería sin embargo el de elevar el déficit en cuenta corriente. En una economía abierta, es interesante entonces notar que el sector externo puede jugar el papel de una válvula de ajuste que permite

$56 \mathrm{Al}$ elevarse $\rho$; se reduce $X$ [véase la ecuación (89)] y se eleva por lo tanto $P_{N}$ [véase (88)] y $P$. Es necesario quizás enfatizar que esta elevación es discreta y no desemboca en una espiral inflacionaria. 
hacer compatibles las peticiones sociales y la productividad de la economía. Un exceso de peticiones puede, por ejemplo, ser "acomodado" mediante una elevación de la deuda externa. ${ }^{57}$

El gobierno puede también querer mantener intacto el equilibrio externo. Lograría este objetivo si redujera (mediante una reducción de la oferta monetaria o una revaluación de la tasa de cambio) el saldo monetario real, en vez de aumentarlo. Esta política restrictiva forzaría sin embargo la economía hacia un equilibrio de depresión (punto $E_{1}$ en la gráfica 3).

El gobierno cuenta finalmente con una última opción que consiste en mantener constante la relación de precios $(X)$. Dada la elevación de $\rho$ en la ecuación (86) esta política conduce a una espiral inflacionaria. La ecuación (88) indica a su vez que una devaluación paralela de la tasa de cambio es requerida para mantener $X$ constante. Y las ecuaciones (91) y (92) señalan finalmente que la oferta monetaria debe también elevarse simultáneamente si se quiere estabilizar el producto y el déficit externo. Esta opción es, por lo tanto, equivalente a la que ya había sido encontrada en el caso de una economía cerrada.

\section{El IMPACTO DE LA POLÍtICA MONETARIA SOBRE PRECIOS, PRODUCTO Y BALANZA DE PAGOS: UNA SÍNTESIS}

El conjunto de modelos que acaba de ser presentado permite sintetizar la esencia del impacto que pueda tener la política monetaria sobre precios, producto y balanza de pagos.

Empezando con el caso del modelo clásico en economía cerrada, se vio que el ajuste instantáneo de precios y salarios permitía conservar en todo instante un sistema de precios relativos y un nivel absoluto de precios que aseguraba equilibrio en todos los mercados. Se alcanzaban, entonces, conclusiones netamente monetaristas: la inflación es un fenómeno monetario y cambios en la oferta monetaria no alteran el producto; repercuten solamente en alzas de precios. Lo más conveniente en materia de política monetaria consiste por lo tanto en dejar la economía equilibrarse por sí sola e inyectar dinero a una tasa moderada que permita asegurar la estabilidad de los precios en un contexto de crecimiento real. Las hipótesis sobre las cuales descansa este modelo son, sin embargo, bastante restrictivas ya que presuponen tanto información como competencia perfectas.

El modelo de los "nuevos clásicos" intenta deshacerse del supuesto de información perfecta y analiza cómo se comportan los agregados económicos cuando las expectativas de los agentes cesan de ser correctas en todo momento. Cambios en la oferta monetaria repercuten entonces temporalmente en ajustes de cantidades (el empleo y el producto), mientras los agentes no hayan corregido sus expectativas. Tan pronto como lo hayan hecho, la actividad económica regresa a su

57 En este modelo no se han introducido impuestos ni términos de intercambio. Pero el impacto de un empeoramiento en los términos de intercambio o de un alza en los impuestos sería parecido al de un incremento en el margen de ganancia o en el salario real. En el primer caso es el sector externo el que está elevando sus "peticiones" sobre el ingreso nacional; en el segundo caso, es el gobierno. 
nivel original y todo el impacto se canaliza de nuevo hacia los precios. La única política monetaria recomendable en el largo plazo es por lo tanto la misma que en el caso anterior. Sólo que en este nuevo contexto, resulta aún más importante hacer crecer la oferta monetaria a un ritmo constante (o por lo menos previsible) para no sorprender al público y evitar de esta manera fluctuaciones innecesarias del producto.

La crítica keynesiana a estos enfoques clásicos enfatiza el impacto de la información imperfecta sobre la capacidad de ajuste de los precios y salarios. Se argumenta que la existencia de información imperfecta origina costos de ajuste importantes que tienden a impedir o a frenar, dentro de cierto margen, los ajustes que serían necesarios para un constante equilibrio. El análisis se vuelve de desequilibrio y se concluye que movimientos moderados de la oferta monetaria no alteran los precios sino que se reflejan más bien en cambios en las cantidades producidas y transactadas. La relación dinero-precios se torna entonces muy difusa: el dinero afecta a los precios sólo cuando se trata de cambios en la oferta monetaria lo suficientemente fuertes como para empujar a la economía fuera de su zona de inercia. Se invierten así las conclusiones del análisis clásico y la intervención monetaria del gobierno se vuelve deseable para estabilizar el producto alrededor del pleno empleo.

El modelo sociológico rechaza, por su parte, la hipótesis de competencia perfecta y adopta como punto de partida una situación de rigidez en las peticiones sobre el ingreso formuladas por los diferentes grupos o sectores sociales que componen a la economía. La inflación surge, entonces, de una incompatibilidad entre la suma de las peticiones y la capacidad de oferta (productividad) del conjunto económico. ${ }^{58}$ En la medida en que el gobierno busque estabilizar el ingreso, la política monetaria se vuelve entonces prisionera del fenómeno sociológico: para evitar la recesión, el dinero tiene que seguir a los precios, y ya no los precios al dinero como en el caso clásico.

Estos diferentes enfoques y modelos fueron ampliados posteriormente para tomar en cuenta el caso de una economía abierta. En el modelo clásico, se encontró que el único factor que determinará el producto y la balanza de pagos era el saldo monetario real, calculado en base al nivel de precios internacional ajustado por la tasa de cambio; de ahí su calificativo de enfoque "monetario" de la balanza de pagos. Se llegó en este caso a la conclusión de que el comportamiento de los agregados dependía de la política seguida por las autoridades monetarias en el manejo del saldo monetario real y de la tasa de cambio. Una política que consistiera en mantener constante el saldo monetario real y en compensar las variaciones en los precios internacionales mediante ajustes inversos en la tasa de cambio permitiría por ejemplo estabilizar simultáneamente los precios, el producto y la balanza de pagos. El gobierno cuenta siempre, no obstante, con otra opción que consiste en expandir más rápidamente la oferta monetaria y dejar que el aumento correspondiente en el déficit externo (acompañado por

58 Si la función de producción es lineal, una espiral inflacionaria es la única salida posible para armonizar el conflicto existente entre peticiones y productividad. Si tiene retornos decrecientes, la inflación sigue siendo aún inevitable si el gobierno insiste en estabilizar el producto alrededor de un nivel dado de pleno empleo. 
un cambio estructural en la composición del producto) absorba una parte de las presiones inflacionarias así generadas.

El análisis keynesiano propone, por su parte, un modelo en el cual los precios y los salarios no son enteramente flexibles y concluye que tanto el saldo monetario real como la tasa de cambio son instrumentos que pueden ser usados en forma independiente para alterar el producto y la balanza de pagos. La obtención de un objetivo determinado de equilibrio interno y externo requiere, por lo tanto, del manejo de ambos instrumentos. $Y$ los precios siguen en parte su inercia propia y en parte los movimientos en los precios internacionales, sin ser jamás afectados, por lo menos dentro de cierto rango, por movimientos en la oferta monetaria.

Finalmente, el enfoque sociológico desemboca en un modelo en el cual los equilibrios interno y externo siguen siendo función del saldo monetario real pero ya no dependen de la tasa de cambio (de ahí el calificativo de "estructuralista"), sino del valor de las peticiones sociales y de la capacidad de oferta de la economía. El déficit en cuenta corriente y el nivel de actividad funcionan, entonces, como dos válvulas alternativas que permiten aliviar las contradicciones existentes entre peticiones y productividad, evitando que éstas se derramen solamente hacia los precios.

La diversidad de los resultados obtenidos obliga así a cuestionar seriamente la naturaleza de los supuestos adoptados y a tratar de analizar empíricamente en profundidad en cual de estos modelos o enfoques es deseable ubicar cada época o fase específica de una economía en particular. Este trabajo es aún más delicado dado que la frontera entre enfoques puede ser, en ciertos casos, solamente una de grado en la magnitud de las oscilaciones: una economía de características esencialmente keynesianas o sociológicas puede, por ejemplo, responder en forma clásica a una desviación muy brutal de la oferta monetaria que obligue a los agentes a revisar rápidamente sus precios o peticiones. Este requerimiento de identificación del modelo subyacente resulta ser por lo tanto un problema muy importante para el econometrista pero constituye una tarea indispensable si se quieren alcanzar conclusiones que tengan algún grado de solidez.

\section{Bibliografía}

ACKLEy, G. (1959) “Administered Prices and the inflationary Process” American Economic Review (proceedings) Mayo.

ANDO, A. (1978) "On a Theoretical and Empirical Basis of Macroeconometric Models" Documento de trabajo, University of Pennsylvania.

Aujac, H. (1950) "L'Inflation. Consequence Momentanée du Comportement des Groupes Sociaux" Economie Appliquee, 1950 No. 4.

Aukrust, O. (1970) "PRIM 1: A model of the Price and Income Distribution Mechanism of an open Economy" Rev. Income Wealth 16 (1).

AzARIADIS, C. (1975) "Implicit Contracts and Under-employment Equilibria" Journal of Political Economy vol. 83. 
BAILY, M.N. (1974) "Wages and Employment Under Uncertain Demand" Review of Economic Studies, vol. 41.

BAILY, M.N. (1977) "On the theory of Layoffs and Unemployment" Econométrica, vol. 45.

BARRo, R.J. y Grossman H.I. (1971) "A General Disequilibrium Model of Income and Employment" American Economic Review, vol. 61 (1).

BARro, R.J. (1979a) "Second Thoughts on Keynesian Economics" American Economic Review, vol. 69 (Mayo).

"The Equilibrium Approach to Business Cycles" University of RochesterDocumento de trabajo (1979b).

BENASSY, J.P. (1975) "Neo-Keynesian Disequilibrium Theory in a Monetary Economy" Review of Economic Studies vol. 42 (4).

"Cost and Demand Inflation Revisited: a Neo-Keynesian Approach" Economie Appliquée, No. 1 (1978).

"Developments in Non-Walrasian Economics and the Microeconomic Foundations of Macroeconomics" Documento de Trabajo-CEPREMAP (1980).

BenAssy, J.P., BOYER R. y GelPI R.M. (1979) "Régulation des Economies Capitalistes et Inflation" Revue Economique (Mayo).

BlaCk, S. (1973) International Money Market and Flexible Exchange Rates. Princeton Studies in International Finance.

BuIss, C. (1981) Notas mimeografiadas sobre algunas controversias recientes en macroeconomía.

BOYER, R. y MISTRAL J. (1978) Accumulation, Inflation et Crises París: Presses Universitaires de France.

Boyer, R. (1979) "Wage Formation in Historical Perspectiva: the French Experience" Cambridge Journal of Economics, vol. 3. 1979.

BRANSON, W. y MYhRMAN J. (1976) "Inflation in open Economies: SupplyDetermined versus Demand-Determined Models" en Inflation in Small Countries (H. Frisch, ed. New-York: Springer-Verlag.

BRECHLING, F.P. R. (1973) "Wage inflation and the Structure of Regional Unemployment" Journal of Money, Credit and Banking, vol. 5 (1).

Bronfenbrenner, M. (1950) "Trade Unions, Pull Employment and Inflation: a Comment", American Economic Review, 40.

Bronfenbrenner, M. y Holzman F.D. (1963) "A Survey of Inflation Theory" American Economic Review, 53 (4).

CARDoso, E. (1979) Inflation, Growth and the Real Exchange Rate: Essays on Economic History in Brazil. Tesis de doctorado. Massachusetts Institute of Technology.

Clower, R.W. (1965) “The Keynesian Counterrevolution: a Theoretical appraisal" The Theory of Interest Rates (Ed. F.H. Hahn and F.P.R. Brechling) London: Macmillan.

DAvidson, P. (1972) Money and the Real World. New-York: Wiley and Sons.

DixIT, A. (1978) "The Balance of Payments in a Model of Temporary Equilibrium with Quantity Rationing" Review of Economic Studies, vol. 45.

Dornbusch, R. (1973) "Money, Devaluation and Nontraded Goods" American Economic Review, vol 63.

Dornbusch, R. (1979) "Exchange Rate Policy and Macroeconomic Stability". 
En The Crawling Peg: Future Prospects and Past Performance J. Williamson, Ed. New-York: Macmillan. Open Economy Macroeconomics New-York: Basic Books (1980).

DrAzEN, A. (1980) "Recent Developments in Macroeconomic Disequilibrium Theory" Econométrica, vol. 48.

Eatwell, J., Llewellyn J. y TaRling R. (1974) "Money Wage Inflation in Industrial Countries" Review of Economic Studies, 1974, No. 4.

Fisher, I. (1911) The Purchasing Power of Money New-York: Macmillan and Co. Ltd.

Frenkel, J. y JOHNSON H.G. eds. (1976) The Monetary Approach to the Balance of Payments. London: Allen and Unwin.

Friedman, M. (1968) "The Role of Monetary Policy" American Economic Review, vol. 58 (Marzo).

Gordon, R.J. (1981) “Output Fluctuations and Gradual Price Adjustment" Joumal of Economic Literature vol. XIX (junio).

HABERLER, G. (1949) "The Market for Foreign Exchanges and the Stability of the Balance of Payments: A Theoretical Analysis" Kyklos, vol. 3.

HAHN, F.H. (1977a) "Exercises in Conjectural Equilibria" Scandinavian Journal of Economics, 79.

-, "Keynesian Economics and General Equilibrium Thenry: Reflections on Some Current Debates" En The Micro-Foundations of Macroeconomics (G. Harcourt, ed.) London International Economic Association (1977b).

- "On Non-Walrasian Equilibria" Review of Economic Studies, vol. 45. (1978).

-, "Monetarism and Economic Theory" Economica vol. 47 (febrero 1980a).

- , "Unemployment from a Theoretical Viewpoint" Economica vol. 47 (agosto, 1980b).

HALL, R. E. (1980) "Employment Fluctuations and Wage Rigidity" Brooking Papers on Economic Activity 1980,1.

Hansen, A. H. (1953) A Guide to Keynes New-York: McGraw Hill.

HARVEY, "Theories of Inflation" Marxism Today (enero 1977).

Hicks, J. (1937) "Mr. Keynes and the Classics: A Suggested Interpretation" Econometrica, vol. 5 (2).

HiNES, A. G. (1964) "Trade Unions and Wage Inflation in the United Kingdom, 1893-1961" Review of Economic Studies, vol. 31 (3).

Holzman, F. D. (1950) "Income Determination in Open Inflation" The Review of Economics and Statistics, vol. 32.

IzE, A. (1980) "A Model of Inflation and Growth in a Developing Economy" Documento de trabajo, El Colegio de México.

KAHN, R. (1977) "Malinvaud on Keynes" Cambridge Journal of Economics $1977,1$.

Kaldor, N. (1959) "Economic Growth and the Problem of Inflation Part II "Economica, vol. 26 (noviembre).

KALECKI, M. (1954) Theory of Economic Dynamics London: Allen and Unwin.

KANTOR, B. (1979) "Rational Expectations and Economic Thought" Journal of Economic Litterature, vol. 17 (diciembre). 
KEYNES, J. M. (1938) The General Theory of Employment, Interest and Money New-York: Harcourt, Brace Inc.

Kouri, P. K. (1975) Three Essays on Flexible Exchange Rates. Tesis de doctorado, Massachusetts Institute of Technology.

LAIDlER, D. E. W. y PARKIN J. M. (1975) "Inflation: a Survey" Economic Joumal, vol. 85.

LE, Bourva J. (1950) "La Theorie d' Inflation" Economie Appliquée, 1950 No. 5.

LEIJONHUfVud, A. (1968) Keynesian Economics and the Economics of Keynes New-York: Oxford University Press.

LiPSEY, R. G. (1960) "The Relationship Between Unemployment and the Rate of Wage Change in the U. K. 1861-1957: A Further Analysis" Economica, vol. 27 (105).

LuCAS, R. E. (1972) "Expectations and the Neutrality of Money" Journal of Economic Theory, vol. 4.

- "Tobin and Monetarism: A Review Article" Journal of Economic Litterature, vol. XIX (1981).

LuCAS, R.E. y SARgENT T. J. (1980) "Rational Expectations and Econometric Practice" Documento de Trabajo.

MACHLUP, F. (1939) "The Theory of Foreing Exchanges" Economica 6 (24).

Malinvaud, E. (1977) The Theory of Unemployment Reconsidered. Basil Blackwell, Oxford.

Marschak, T. y Selten R. (1974) General Equilibrium with Price Making Firms Berlin: Springerverlag.

- "Restabilizing Responses Inertia Supergames and oligopolistic equilibria" Quarterly Journal of Economics, 92 (1) (1978).

Marshall, A. (1920) Principles of Economics London: Macmillan and Co. Ltd.

MAYNARD, G. y VAN RYckeghem W. (1976) A World of Inflation London: Batsford.

Mccallum, B. T. (1979) “The Current State of the Policy Ineffectiveness Debate" American Economic Review, vol. 69.

- "Hahn's Theoretical Viewpoint on Unemployment: A Comment" Economica, vol. 47. (1980).

-, "Rational Expectations and Macroeconomic Stabilization Policy: An Overview" Journal of Money, Credit and Banking, vol. (12) No. 4. (1980).

MeAde, J. E. (1951) The Balance of Payments London: Oxford University Press.

Modigliani, P. (1977) "The Monetarist Controversy or, Should we Forsake Stabilization Policies?" American Economic Review vol. 67 (Marzo).

Morton, W. A. (1950) "Trade Unions, Full Employment and Inflation" American Economic Review, 40.

MulveY, C. y Trevithick J. A. (1974) "Some Evidence on the Wage Leadership Hypothesis" Scottish Journal of Political Economy, vol. 21 (1).

MuNDELl, R. A. (1961) "The International Disequilibrium System", Kiklos 14 (2).

-, International Economics New-York: Macmillan (1968).

Murh, J. F. (1961) "Rational Expectations and the Theory of Price Movements" Econometrica, vol. 29 (3). 
NeGISHI, T. (1966) “Monopolistic Competition and General Equilibrium” Review of Economic Studies, vol. 28.

- Microeconomic Foundations of Keynesian Macroeconomics Amsterdam: North-Holland (1979).

NikaIdo, H. (1975) Monopolistic Competition and Effective Demand Princeton University Press.

Noyola, J. (1956) "El Desarrollo Económico y la Inflación en México y Otros Países Latinoamericanos" Investigación Económica No. 4.

Okun, A. (1981) Prices and Quantities: A Macroeconomic Analysis Washington: The Brookings Institution.

Olivera, J. (1964) "On Structural Inflation and Latin American Structuralism" Oxford Economic Papers XVI, No. 3.

P Atinkin, D. (1956) Money, Interest and Price. An Integration of Monetary and Value Theory New-York: Harper and Row.

Phelps, E. (1967) "Phillips Curves Expectations of Inflation and Optimal Unemployment over Time" Economica, vol. 34 (agosto).

PhelPS, E. (1981) Okun's Micro-Macro System: A Review Article Journal of Economic Litterature (Septiembre).

Phillirs, A. W. (1958) "The Relationship Between Unemployment and the Rate of Wage Change in the U. K. 1861-1957" Economica, vol. 25 (100).

Pigou, A. C. (1933) Theory of Unemployment. London: Macmillan and Co. Ltd.

P REBISCH, R. (1970) "Structural Vulnerability and Inflation" Leading Issues in Economic Development: Studies in International Poverty (G. M. Meier, editor) New-York: Oxford University Press.

Robinson, J. (1937) "The Foreing Exchanges" en Essays in the Theory of employment New-York: Macmillan.

, Collected Economic Papers Oxford University Press. (1973).

RowTHORN, B. (1980) Capitalism, Conflict and Inflation London: Lawrence and Wishart.

SACHS, J. (1979) 'Wages, Profits and Macroeconomic Adjustment: A Comparative Study" Brooking Papers on Economic Activity, No. 2.

SARgent, T. J. y Wallace N. (1973) "Rational Expectations and the Dynamics of Hyper inflation" International Economic Review, vol. 14 (2).

- _ "Rational Expectations, the Optimal Monetary Instrument and the Optimal Money Supply Rule" Journal of Political Economy, vol. 83 (Abril, 1975).

SChOtTER, A. y Schwodiauer G. (1980) "Economics and the Theory of Games: a Survey" Journal of Economic Litterature, vol. 18 (junio).

Schultze, C. (1959) "Recent Inflation in the United States" Study Paper No. 1 in joint Economic Committee. Washington.

SEERS, D. (1962) "A Theory of Inflation and Growth in Under developed Economics based on the Experience of Latin America" Oxford Economic Papers XIV, No. 2.

Soderstrom, H. T. y Viotti S. (1975) "Prices, Wages and Employment in the Small Open Economy" Documento de Trabajo, Institute for International Economic Studies-Stockholm University.

Solis, L. (1971) "Mexican Economic Policy in the Post-War-Period: The Views 
of Mexican Economists" The American Economic Review (Suplemento) vol. 61 No. 3.

Solow, R.(1980) “On Theories of Unemployment” American Economic Review, 70 (Marzo).

S PINELl, F. (1981) "The Wage Push Hipothesis: The Italian Case" Journal of Monetary Economics. vol. 6 No. 4 (Octubre).

S wan, T. W. (1955) "Longer-Run Problems of the Balance of Payments" The Australian Economy: A Volume of Readings (H. W. Amdt y W. M. Corden, Eds.) Melbourne: Cheshire Press.

TAYLOR, J. (1979) "Staggered Wage Setting in a Macro Model"American Economic Review, papers and proceedings.

THORP, W. y QUANDT R. (1959) The New Inflation New-York: McGraw Hill.

ToBIN, J. (1981) "The Monetarist Counter-Revolution Today-an Appraisal" The Economic Journal vol. 91 (Marzo).

WACHTER, S. (1976) Latin American Inflation. Lexington Books.

Weintraub, S. (1978) Keynes, Keynesians and Monetarists. University of Pennsylvania Press. 